\title{
QUANTITATIVE DARBOUX THEOREMS IN CONTACT GEOMETRY
}

\author{
JOHN B. ETNYRE, RAFAL KOMENDARCZYK, AND PATRICK MASSOT
}

\begin{abstract}
This paper begins the study of relations between Riemannian geometry and contact topology on $(2 n+1)$-manifolds and continues this study on 3-manifolds. Specifically we provide a lower bound for the radius of a geodesic ball in a contact $(2 n+1)$-manifold $(M, \xi)$ that can be embedded in the standard contact structure on $\mathbb{R}^{2 n+1}$, that is, on the size of a Darboux ball. The bound is established with respect to a Riemannian metric compatible with an associated contact form $\alpha$ for $\xi$. In dimension 3, this further leads us to an estimate of the size for a standard neighborhood of a closed Reeb orbit. The main tools are classical comparison theorems in Riemannian geometry. In the same context, we also use holomorphic curve techniques to provide a lower bound for the radius of a PS-tight ball.
\end{abstract}

\section{INTRODUCTION}

Darboux's theorem in contact geometry says that any point in a $(2 n+1)-$ dimensional contact manifold has a neighborhood that can be identified with an open ball in $\mathbb{R}^{2 n+1}$ with its standard contact structure. In [8] it was shown that a quantitative version of Darboux's theorem can give interesting global information about a contact structure on a 3-manifold. For example one can give Riemannian geometric criteria for a contact structure to be universally tight and in addition prove a contact geometric version of the sphere theorem. Such results rely on deep theorems about contact 3-manifolds. Our understanding of contact manifold in higher dimensions is much less advanced, but we will still be able to prove a quantitative version of Darboux's theorem.

Given a contact structure $\xi$ on a $(2 n+1)$-manifold $M$ and a Riemannian metric $g$ we can define the Darboux radius of $(\xi, g)$ at a point $p \in M$ as

$$
\begin{aligned}
\delta_{p}(\xi, g)=\sup \{r & <\operatorname{inj}(g, p) \mid \text { the open geodesic ball }\left(B_{p}(r), \xi\right) \text { at } p \text { of radius } r \text { is } \\
& \text { contactomorphic to an open subset in } \left.\left(\mathbb{R}^{2 n+1}, \xi_{\text {std }}\right)\right\}
\end{aligned}
$$

(where $\operatorname{inj}(g, p)$ is the injectivity radius of $g$ at $p$ ) and the Darboux radius of $(\xi, g)$ to be

$$
\delta(\xi, g)=\inf _{p \in M} \delta_{p}(\xi, g) .
$$

One would like to estimate these quantities in terms of $g$. In dimension 3 this was done in [8] but relied heavily on a theorem of Eliashberg [7] that says that any tight contact structure on a 3 -ball is embeddable in the standard contact structure on $\mathbb{R}^{3}$. In particular in [8] we defined the tightness radius at a point $p, \tau_{p}$, and the tightness radius $\tau$ for a contact metric 3-manifold. Eliashberg's theorem identifies

Received by the editors September 17, 2012 and, in revised form, January 9, 2015.

2010 Mathematics Subject Classification. Primary 53D10, 53D35; Secondary 57R17. 
$\tau_{p}$ and $\tau$ with $\delta_{p}$ and $\delta$, respectively. As Eliashberg's theorem is unavailable to us in higher dimensions and it is even plausible that it does not hold, we will discuss direct arguments to estimate $\delta$.

But first, a more direct generalization of the estimates proved in [8] would be to estimate the size of geodesic balls in contact manifolds that were in some sense "tight". We say a contact structure $\xi$ on $M^{2 n+1}$ is PS-overtwisted if it contains a bLob (see Section 3.3 for the relevant definitions). Otherwise we say $\xi$ is $P S$-tight. Given a contact $(2 n+1)$-manifold $(M, \xi)$ and a Riemannian metric $g$ we now define the $P S$-tightness radius at $p \in M$ with respect to $g$ to be

$$
\begin{gathered}
\tau_{p}^{\mathrm{PS}}(\xi, g)=\sup \left\{r<\operatorname{inj}(g, p) \mid \text { the geodesic ball } B_{p}(r) \text { at } p\right. \\
\text { of radius } r \text { is PS-tight }\},
\end{gathered}
$$

and the $P S$-tightness radius of $M$ to be

$$
\tau^{\mathrm{PS}}(\xi, g)=\inf _{p \in M} \tau_{p}^{\mathrm{PS}}(\xi, g) .
$$

After this paper was written, Borman, Eliashberg and Murphy [4] defined a notion of an "overtwisted disk" in all dimensions and proved that contact structures containing such a disk satisfy an $h$-principle. In particular, all almost contact structures are homotopic to contact structures with an overtwisted disk and any two such contact structures that are homotopic through almost contact structures are isotopic. So this notion of overtwisted is "the right one" in higher dimensions, and we can use it to define the tightness radius $\tau(\xi, g)$. It is proved in [14, 15] that the standard structure on $\mathbb{R}^{2 n+1}$ is PS-tight and in [4] that an overtwisted contact structure is PS-overtwisted so $\delta(\xi, g) \leq \tau^{\mathrm{PS}}(\xi, g) \leq \tau(\xi, g)$. The relation between tightness and PS-tightness is not yet completely elucidated and they may be equivalent. In any case, all our results about $\tau^{\mathrm{PS}}(\xi, g)$ (which are lower bounds) also hold equally well for $\tau(\xi, g)$.

Again, in dimension 3 the above quantities all are equal:

$$
\tau^{\mathrm{PS}}(\xi, g)=\tau(\xi, g)=\delta(\xi, g),
$$

because in that dimension $P S$-tight is equivalent to tight, which, for a 3 -ball, in turn is equivalent to being a Darboux ball. In higher dimensions it is not known whether $\tau^{\mathrm{PS}}(\xi, g)$ can be strictly larger than $\delta(\xi, g)$.

1.1. Estimates for $\tau^{\mathrm{PS}}$. The first result in this article extends convexity type estimates for $\tau^{\mathrm{PS}}(M, \xi)$ from [8] to higher dimensions in the setting of compatible metrics. In higher dimensions the definition of a metric $g$ being compatible with a contact structure $\xi$ on $M^{2 n+1}$ is more complicated than the one considered in [8] for dimension 3. We refer to Definition 2.7 for the precise details. Here we merely note that given a contact form $\alpha$ for $\xi$ used in the definition of compatibility, Proposition 2.3 gives a complex structure $J$ on $\xi$, and the metric can be written as

$$
g(v, w)=\frac{1}{\theta^{\prime}} d \alpha\left(v, J w^{\xi}\right)+\alpha(v) \alpha(w),
$$

where $\theta^{\prime}$ is constant and measures the instantaneous rotation speed of $\xi$ (see Remark 2.4), and $w^{\xi}$ denotes the component of the vector $w$ lying in the $\xi$ component of the splitting of $T M$ into $\xi$ and the span of the Reeb vector field of $\alpha$. 
We obtain the following generalization of Theorem 1.3 from [8].

Theorem 1.1. Let $\left(M^{2 n+1}, \xi\right)$ be a contact manifold and $(\alpha, g, J)$ be a compatible metric structure for $\xi$. Then,

$$
\tau^{\mathrm{PS}}(\xi, g) \geq \operatorname{conv}(g)
$$

where

$$
\begin{gathered}
\operatorname{conv}(g)=\sup \{r \mid r<\operatorname{inj}(g) \text { and the geodesic spheres of radius } r \\
\text { are geodesically convex }\}
\end{gathered}
$$

and $\operatorname{inj}(g)$ is the injectivity radius of $(M, g)$. In particular, if $\sec (g) \leq K$, for $K>0$, then

$$
\tau^{\mathrm{PS}}(\xi, g) \geq \min \left\{\operatorname{inj}(g), \frac{\pi}{2 \sqrt{K}}\right\}
$$

and $\tau^{\mathrm{PS}}(\xi, g)=\operatorname{inj}(g)$ if $g$ has non-positive curvature.

As in dimension 3 the bound on $\tau^{\mathrm{PS}}$ is especially effective in the case of nonpositive curvature.

Corollary 1.2. Let $(M, \xi)$ be a $(2 n+1)$-contact manifold and $g$ a complete Riemannian metric compatible with $\xi$ having non-positive sectional curvature. Then $\xi$ pulled back to the universal cover of $M$ is PS-tight. In particular, if $(M, \xi)$ contains a bLob $N$, then the image of $i_{*}: \pi_{1}(N) \rightarrow \pi_{1}(M)$, where $i: N \rightarrow M$ is the inclusion map, is infinite.

Remark 1.3. Sasakian (or, more generally, $K$-contact) manifolds are contact metric manifolds satisfying some extra conditions; see [3]. One can naturally wonder whether Theorem 1.1 is relevant to their study. But those manifolds do not contain any bLob since one can combine the main results of [16] and [19] to prove that they are symplectically fillable. The same conclusion actually holds for the wider class of integrable $C R$-contact metric manifolds in dimension at least 5 ; see Definition 2.8 . As explained in [6, Theorem 5.60] this can be proved using deep results in complex analysis due to Lempert, Hironaka and Rossi. However, Theorem 1.5 below still gives non-trivial information in this context.

Remark 1.4. Recall that in dimension 3 a contact structure is called universally tight if the contact structure is tight when pulled back to the universal cover and virtually overtwisted if it is overtwisted when pulled back to a finite cover. Since an overtwisted disk is contractible it can be lifted to any cover; thus if a contact structure is universally tight, then it is also tight. Since the fundamental groups of 3-manifolds are residually finite it is also known that being universally tight is equivalent to not being virtually overtwisted. One can make the same definitions in higher dimensions for PS-universally tight and PS-virtually overtwisted and similarly for universally tight and virtually overtwisted using the Borman-EliashbergMurphy notion of overtwisted. Since their notion of overtwisted also involves disks one again sees that universally tight implies not virtually overtwisted, which in turn implies tight, but now we do not know if universally tight is equivalent to not virtually overtwisted (though it seems unlikely due to the fact that fundamental groups in higher dimensions need not be residually finite). Note in particular that Corollary 1.2 implies that $(M, \xi)$ is universally tight. The relation between PS-universally tight and PS-virtually overtwisted and PS-tight is much more complicated due to the fact that a bLob need not be simply connected and hence it 
might not lift to covers. Notice that the condition in Corollary 1.2 on the fundamental group of a bLob implies that its preimage in the universal cover of $M$ will be non-compact and hence not a bLob.

1.2. Direct geometric methods for estimating the Darboux radius. We now discuss a method for estimating $\delta(\xi, g)$ in higher dimensions. This strategy is more geometric and direct than the one used in [8] to bound the Darboux radius in dimension 3, as it does not use holomorphic curves or classification results. We want a control on the Darboux radius using control on curvature, the rotation speed $\theta^{\prime}$ and the tensor $[J, J]$ (the latter measures how far the induced CR structure is from being integrable; see Section 2 for definitions). Note that if $K$ is a bound on sectional curvature, then $1 / \theta^{\prime},\|[J, J]\|$ and $1 / \sqrt{K}$ behave like lengths under homothety (constant rescaling of the metric). This explains the appearance of such terms in the following estimate. It is also expected that the estimate deteriorates when the rotation speed increases or when one widens the sectional curvature interval but also when $\|[J, J]\|$ increases since compatible metrics are less tied to the contact structure for non-integrable CR structures.

Theorem 1.5. Let $\left(M^{2 n+1}, \xi\right)$ be a $(2 n+1)$-dimensional contact manifold and $(\alpha, g, J)$ be a complete compatible metric structure for $\xi$ with rotation speed $\theta^{\prime}$. If the sectional curvature of $g$ is contained in the interval $[-K, K]$ for some positive $K$, then

$$
\delta(\xi, g) \geq \min \left(\frac{\operatorname{inj}(g)}{2}, \frac{1}{208 n^{2} \max \left(\sqrt{K},\|[J, J]\|, \theta^{\prime}\right)}\right),
$$

where $[J, J]$ is the Nijenhuis torsion of the complex structure $J$ on $\xi$.

Theorem 4.1 is a better, but more technical, bound on $\delta(\xi, g)$ and is proven in Section 4 after the explicit quantities used in the better estimate are explained, but the main point is the existence of an explicit bound rather than its precise expression.

Remark 1.6. Since every contact structure has a compatible metric structure, this theorem always produces an explicit bound on the Darboux radius in terms of Riemannian curvature information.

In dimension 3 we can improve the bound on $\delta(\xi, g)$ coming from these geometric methods.

Theorem 1.7. Let $(M, \xi)$ be a contact 3-manifold and $g$ a complete Riemannian metric that is compatible with $\xi$ and has rotation speed $\theta^{\prime}$. Then,

$$
\delta(\xi, g)=\tau(M, \xi) \geq \min \left\{\frac{\operatorname{inj}(g)}{2}, \frac{\pi}{2 \sqrt{K}}, \frac{2}{\sqrt{2 A+B^{2}}+B}\right\},
$$

where

$$
A=\frac{4}{3}|\sec (g)|, \quad B=\frac{\theta^{\prime}}{2}+\sqrt{\frac{\left(\theta^{\prime}\right)^{2}}{4}-\frac{1}{2} \min _{p \in M}\left(\operatorname{Ric}_{p}\left(R_{\alpha}\right)\right)}
$$

and Ric is the Ricci tensor, $R_{\alpha}$ is the Reeb vector field of $\alpha$ and $|\sec (g)|$ is the maximum in absolute value of the sectional curvature over $(M, g)$, and $K$ is an upper bound for the sectional curvature. 
Theorems 1.5 and 1.7 provide bounds on $\delta(\xi, g)$ which are weaker than the one given in Theorem 1.1 in dimension 3. But the ideas underlying their proof extend to higher dimensions and can be used when both the convexity of the boundary assumption of Theorem 1.1 and the absence of closed Reeb orbits fail, thus ruining the strategy used to prove Theorem 1.1. As an example of such a situation we show the following estimate on the size of a standard neighborhood of a closed Reeb orbit in a contact 3 -manifold.

Theorem 1.8. Let $(M, \xi)$ be a contact 3-manifold and $g$ a complete Riemannian metric that is compatible with $\xi$. Let $\gamma$ be a closed Reeb orbit and $\mathrm{T}(r)$ an embedded geodesic tube of radius $r$ about $\gamma$. If $r$ is below the bound of Equation (1.2), then the contact structure restricted to $\mathrm{T}(r)$ is universally tight and, moreover, can be embedded in $\left(S^{1} \times \mathbb{R}^{2}, \xi_{\text {std }}=\operatorname{ker}\left(d \phi+r^{2} d \theta\right)\right)$.

1.3. Outline. In Section 2 we define the notions of compatibility between metrics and contact structures in an arbitrary dimension. The paper then splits into two logically independent threads which both depend on Section 2 . The first one is covered in Section 3, which compares Riemannian and almost complex convexity and proves Theorem 1.1 and Corollary 1.2. The second one starts in Section 4 which states the refined version of Theorem 1.5 and proves it modulo a number of propositions which are proved in Subsections 4.1 to 4.4. Section 5] explains how geometrical methods of Section 4 can be strengthened using topological methods which are specific to dimension 3 and proves Theorems 1.7 and 1.8 .

\section{Metrics compatible with contact structures}

Throughout this section and the rest of the paper we will interchangeably use the notation $g(u, v)$ and $\langle u, v\rangle$ to denote the Riemannian metric evaluated on the vectors $u$ and $v$.

On a vector space $V$, let $\omega$ be a symplectic pairing, $g$ an inner product and $J$ a complex structure. Recall that $(g, \omega, J)$ is called a compatible triple if $g(\cdot, \cdot)=$ $\omega(\cdot, J \cdot)$. In particular $J$ is tamed by $\omega$, that is, $\omega(v, J v)>0$ for $v \neq 0$, and we also have $\omega(J u, J v)=\omega(u, v)$. Note that two members of the triple uniquely determine the third one. We say that $g$ and $\omega$ are compatible if there exists some (unique) $J$ such that $(g, \omega, J)$ is a compatible triple.

In this paper we say that $g$ and $\omega$ are weakly compatible if there is a positive number $c$ such that $c g$ and $\omega$ are compatible. In that case, volume forms induced by $c g$ and $\omega$ on $V$ are equal; hence $c$ and then $J$ are uniquely determined by $\omega$ and $g$.

Definition 2.1. We say a contact structure $\xi$ and a Riemannian metric $g$ on a $(2 n+1)$-manifold $M$ are weakly compatible if there is a contact form $\alpha$ for $\xi$ such that its Reeb vector field $R_{\alpha}$ is orthogonal to $\xi$ and $\left.g\right|_{\xi}$ and $\left.(d \alpha)\right|_{\xi}$ are, pointwise, weakly compatible.

We note right away that the contact form involved in the above definition is unique up to a constant multiple because of the following well-known lemma.

Lemma 2.2. Two contact forms on a connected manifold which have the same kernel and parallel Reeb fields are constant multiples of each other.

Proof. Given two such forms $\alpha$ and $\alpha^{\prime}$, the kernel condition means there is some positive function $f$ such that $\alpha^{\prime}=f \alpha$. By Darboux's theorem for contact forms, 
each point lies in a coordinate chart where $\alpha=d z-\sum y_{i} d x_{i}$ and its Reeb field is $\partial_{z}$. We can compute:

$$
\iota_{\partial_{z}} d \alpha^{\prime}=-\sum\left(y_{i} \partial_{z} f+\partial_{x_{i}} f\right) d x_{i}-\sum \partial_{y_{i}} f d y_{i} .
$$

So the Reeb field condition is equivalent to $\partial_{y_{i}} f=0$ and $y_{i} \partial_{z} f+\partial_{x_{i}} f=0$ for all $i$. Differentiating the second equation with respect to $y_{i}$ one sees that $\partial_{z} f=0$ and then that $\partial_{x_{i}} f=0$ for all $i$. Thus $d f=0$ and $f$ is constant.

One then has the following mostly tautological proposition.

Proposition 2.3. Let $\xi$ be a contact structure on $a(2 n+1)-$ manifold $M$ and $g$ a weakly compatible metric. Then fixing a contact form $\alpha$ as in the definition of weakly compatible we denote by $c$ the positive function and $J$ the complex structure on $\xi$ such that $\left(\left.c g\right|_{\xi},\left.d \alpha\right|_{\xi}, J\right)$ is a compatible triple on $\xi$.

The complex structure $J$ can be extended to a linear map on TM as follows:

$$
\phi: T M \rightarrow T M: v \rightarrow J v^{\xi},
$$

where

$$
v^{\xi}=v-g(v, n) n
$$

is the component of $v$ lying in $\xi$ (here $n=R_{\alpha} /\left\|R_{\alpha}\right\|$ is the unit normal vector to $\xi)$. The metric $g$ may then be expressed as

$$
g(u, v)=\frac{\rho}{\theta^{\prime}} d \alpha(u, \phi(v))+\rho^{2} \alpha(u) \alpha(v),
$$

where $\rho=\left\|R_{\alpha}\right\|$ and $\theta^{\prime}=\rho c$.

Remark 2.4. Our choice of notation $\theta^{\prime}$ in the above proposition can be explained as follows. Let $\xi$ be a cooriented hyperplane field on any Riemannian manifold $(M, g)$. Let $n$ be the positive unit normal vector field of $\xi$ and let $\beta=g(n, \cdot)$ be the unit 1form defining $\xi$. The restriction of $d \beta$ to $\xi$ measures how fast $\xi$ rotates along vector fields tangent to $\xi$. Indeed if $X$ and $Y$ are two such vector fields with unit norm and $\varphi_{t}$ is the flow of $X$, then the derivative at $t=0$ of the angle between $n$ and $\varphi_{t}^{*} Y$ is $d \beta(X, Y)$ (this results from a short computation). If $\xi$ is a contact structure and $g$ is weakly compatible with $\xi$, then equation (2.3) and a short computation prove that $\theta^{\prime}=d \beta(u, J u)$ for any unit vector $u$ in $\xi$. Hence $\theta^{\prime}$ is indeed the derivative of some angle.

Remark 2.5. It is useful to notice that $\phi^{2}$ can be expressed as

$$
\phi^{2}(v)=-v+\alpha(v) R_{\alpha}
$$

for all vectors $v \in T M$.

It is difficult to say much about weakly compatible metrics in higher dimensions, but if we assume the length of $R_{\alpha}$ is constant, then we can understand something about the covariant derivatives of vectors with respect to $R_{\alpha}$ and observe that the flow of the Reeb vector field traces out geodesics.

Proposition 2.6. Let $g$ be a metric weakly compatible with the contact structure $\xi$ on the $(2 n+1)$-manifold $M$. Let $R_{\alpha}$ be the Reeb vector field associated to the form $\alpha$ implicated in the definition of $g$ being weakly compatible with $\xi$. If $\rho=\left\|R_{\alpha}\right\|$ is constant, then

$$
\nabla_{R_{\alpha}} R_{\alpha}=0
$$


In particular, flow lines of $R_{\alpha}$ are geodesics. Moreover, if $v$ is a vector field tangent to $\xi$, then $\nabla_{R_{\alpha}} v$ is also tangent to $\xi$.

Proof. We first notice that $\alpha$ and $R_{\alpha}$ are related by

$$
\rho^{2} \alpha=\iota_{R_{\alpha}} g,
$$

since they agree on $\xi$ and on $R_{\alpha}$. For any vector field $v$ the definition of the Reeb vector field implies $d \alpha\left(R_{\alpha}, v\right)=0$. On the other hand, using the assumption that $\rho$ is (a non-zero) constant, we compute

$$
\begin{aligned}
d \alpha\left(R_{\alpha}, v\right) & =R_{\alpha} \cdot \alpha(v)-v \cdot \alpha\left(R_{\alpha}\right)-\alpha\left(\left[R_{\alpha}, v\right]\right) \\
& =R_{\alpha} \cdot \alpha(v)-\alpha\left(\nabla_{R_{\alpha}} v-\nabla_{v} R_{\alpha}\right) \\
& =\rho^{-2}\left(R_{\alpha} \cdot\left\langle R_{\alpha}, v\right\rangle-\left\langle R_{\alpha}, \nabla_{R_{\alpha}} v-\nabla_{v} R_{\alpha}\right\rangle\right) \\
& =\rho^{-2}\left(\left\langle\nabla_{R_{\alpha}} R_{\alpha}, v\right\rangle+\left\langle R_{\alpha}, \nabla_{R_{\alpha}} v\right\rangle-\left\langle R_{\alpha}, \nabla_{R_{\alpha}} v\right\rangle+\left\langle R_{\alpha}, \nabla_{v} R_{\alpha}\right\rangle\right) \\
& =\rho^{-2}\left(\left\langle\nabla_{R_{\alpha}} R_{\alpha}, v\right\rangle\right),
\end{aligned}
$$

where the last equality follows since $2\left\langle R_{\alpha}, \nabla_{v} R_{\alpha}\right\rangle=v \cdot\left\langle R_{\alpha}, R_{\alpha}\right\rangle=0$. So $\left\langle\nabla_{R_{\alpha}} R_{\alpha}, v\right\rangle$ $=0$ for every $v$ and thus $\nabla_{R_{\alpha}} R_{\alpha}=0$.

If $v$ is a vector field tangent to $\xi$, then

$$
\left\langle R_{\alpha}, \nabla_{R_{\alpha}} v\right\rangle=\left\langle\nabla_{R_{\alpha}} R_{\alpha}, v\right\rangle+\left\langle R_{\alpha}, \nabla_{R_{\alpha}} v\right\rangle=R_{\alpha} \cdot\left\langle R_{\alpha}, v\right\rangle=0,
$$

so $\nabla_{R_{\alpha}} v$ is tangent to $\xi$.

We will not say more about weak compatibility in higher dimensions and restrict our attention to the stronger notion of compatibility.

Definition 2.7. We say a contact structure $\xi$ and a Riemannian metric $g$ on a $(2 n+1)$-manifold $M$ are compatible if there is a contact form $\alpha$ for $\xi$ such that its Reeb vector field $R_{\alpha}$ is orthogonal to $\xi$, has unit length $\left\|R_{\alpha}\right\|=1$, and $\left.g\right|_{\xi}$ and $\left.(d \alpha)\right|_{\xi}$ are, pointwise, weakly compatible and the function $\theta^{\prime}$, defined in Proposition 2.3. is constant.

Equivalently, we can say $g$ and $\xi$ are compatible if there is a contact form $\alpha$ for $\xi$ and a complex structure $J$ on $\xi$ that is compatible with $d \alpha$ on $\xi$, satisfies

$$
g(u, v)=\frac{1}{\theta^{\prime}} d \alpha(u, J v),
$$

for all $u, v \in \xi$, where $\theta^{\prime}$ is some positive constant and for which the Reeb vector field $R_{\alpha}$ is the unit normal to $\xi$.

Recall [3, p. 63] that the Nijenhuis torsion $[T, T]$ of a $(1,1)$-tensor field $T$ is a skew-symmetric tensor field of type $(1,2)$ defined as

$$
[T, T](X, Y)=T^{2}[X, Y]+[T X, T Y]-T[T X, Y]-T[X, T Y] .
$$

Definition 2.8. Let $\xi$ be a (cooriented) contact structure on a $(2 n+1)$-manifold $M$ and $\alpha$ a contact form for $\xi$. A complex structure $J$ on $\xi$ is called a $C R$-structure (or more specifically a strictly pseudo-convex integrable $C R$-structure) if and only if $J$ is tamed by $d \alpha$ and the Nijenhuis torsion $[J, J]$ of $J$ on $\xi$ vanishes. The latter explicitly says that for all sections $v$ and $w$ of $\xi$,

$$
[J, J](v, w)=-[v, w]+[J v, J w]-J([J v, w]+[v, J w])=0 .
$$


Notice that $[J, J]$ is well defined as a $(1,2)$-tensor field on $\xi$ because

$$
\alpha([J v, w]+[v, J w])=-d \alpha(J v, w)-d \alpha(v, J w)=-d \alpha(J v, w)+d \alpha(J v, w)=0 .
$$

Thus

$$
[J v, w]+[v, J w] \text { is tangent to } \xi .
$$

Similarly one can see that $[J v, J w]-[v, w]$ is tangent to $\xi$.

After [3], we call $(M, \alpha, g, J)$ a contact metric structure whenever $\alpha$ and $J$ define the compatible metric $g$ as above. Equivalently we say that $(\alpha, g, J)$ is a compatible metric structure on $(M, \xi)$. Additionally, if $J$ and $\xi$ define a $C R$-structure we say that $(M, \alpha, g, J)$ is a $C R$-contact metric structure.

Note that the above integrability condition is automatic for any complex structure on a plane field in dimension 3. Indeed, we may choose a (local) basis $\{v, \phi(v)\}$ of $\xi$ and observe that

$$
[J, J](v, v)=0 \quad \text { and } \quad[J, J](v, J v)=0,
$$

from which equation (2.9) follows.

We are now ready to state relations between the various operators and their derivatives. Most of these formulas are well known in the literature ( $c f$. [3] ), though extra terms occur due to the generality we are considering here. We also note that there are some sign discrepancies with [3], coming from the fact that we are always using positive contact structures. Due to this, and for the convenience of the reader, we provide proofs of these formulas here.

Recall that the second fundamental form $I I$ of $\xi$ is the quadratic form on $\xi$ defined as follows [18]: for vectors $u$ and $v$ in $\xi_{p}=T_{p} M \cap \xi$,

$$
I I(u, v)=\frac{1}{2}\left\langle\nabla_{u} v+\nabla_{v} u, R_{\alpha}\right\rangle .
$$

We also define the endomorphism

$$
h=\frac{1}{2} \mathcal{L}_{R_{\alpha}} \phi: T M \rightarrow T M,
$$

where $\mathcal{L}$ denotes the Lie derivative. This endomorphism is a repackaging of $I I$ (computations from the proof of Proposition 2.10 show that $h\left(R_{\alpha}\right)=0$ and, for $u$, $v$ in $\xi, I I(u, v)=\langle u, \phi(h v)\rangle)$.

Lemma 2.9. Let $(\alpha, g, J)$ be a compatible metric structure on $(M, \xi)$. Then $h(T M)$ $\subset \xi$.

Proof. First compute

$$
h(v)=\frac{1}{2}\left(\mathcal{L}_{R_{\alpha}} \phi\right)(v)=\frac{1}{2}\left(\mathcal{L}_{R_{\alpha}}(\phi(v))-\phi\left(\mathcal{L}_{R_{\alpha}} v\right)\right)=\frac{1}{2}\left(\left[R_{\alpha}, \phi(v)\right]-\phi\left(\left[R_{\alpha}, v\right]\right)\right) .
$$

Since for any section $u$ of $\xi$ we have $\alpha\left(\left[R_{\alpha}, u\right]\right)=-d \alpha\left(R_{\alpha}, u\right)=0$, we see that $\left[R_{\alpha}, u\right]$ is in $\xi$ for any vector field $u$ in $\xi$. The result follows since the image of $\phi$ is contained in $\xi$.

The basic relations between metric and contact geometric quantities are derived in the following proposition. 
Proposition 2.10. Let $(\alpha, g, J)$ be a compatible metric structure on the contact manifold $(M, \xi)$. Let $R_{\alpha}$ be the Reeb vector field of $\alpha, h$ the endomorphism defined above and II the second fundamental form of $\xi$. Then the following equations hold:

$$
\begin{aligned}
& \langle h(u), v\rangle=\langle u, h(v)\rangle, \\
& \nabla_{v} R_{\alpha}=\phi\left(\left(\theta^{\prime} / 2\right) v-h(v)\right), \\
& (\phi h+h \phi)(v)=0, \\
& I I(v, v)+I I(J v, J v)=0,
\end{aligned}
$$

and

$$
\nabla_{R_{\alpha}}(J v)=J\left(\nabla_{R_{\alpha}} v\right),
$$

for any $v, u \in \xi$ (in the last equation, $v$ has to be a vector field tangent to $\xi$ ).

We will also need the following relations between curvature and contact geometric properties.

Proposition 2.11. Let $(\alpha, g, J)$ be a compatible metric structure on $(M, \xi)$. Then for any vectors $u, v$ and $w$,

$$
\left\|\nabla R_{\alpha}\right\|=B
$$

and

$$
\left|\left\langle\left(\nabla_{u} \phi\right)(v), w\right\rangle\right| \leq\left(\frac{1}{2}\|[J, J]\|+2 B\right)\|u\| \cdot\|v\| \cdot\|w\|
$$

where

$$
B=\frac{\theta^{\prime}}{2}+\sqrt{\frac{\left(\theta^{\prime}\right)^{2}}{4}-\frac{1}{2} \min _{u \in \xi,\|u\|=1}\left(K\left(u, R_{\alpha}\right)+K\left(J u, R_{\alpha}\right)\right)}
$$

and $K(u, v)$ is the sectional curvature of the plane spanned by the unit vectors $u$ and $v$.

A key to proving the above results is the computation of the covariant derivative of $\phi$.

Lemma 2.12. Let $(\alpha, g, J)$ be a compatible metric structure on $(M, \xi)$. Then for any vectors $u, v$ and $w$ the following equation holds:

$$
\left\langle\left(\nabla_{u} \phi\right)(v), w\right\rangle=\frac{1}{2}\left\langle u^{\xi},[J, J]\left(v^{\xi}, J w^{\xi}\right)+\left(\left(2 h-\theta^{\prime} I d\right) \wedge \alpha\right)(v, w)\right\rangle
$$

where, for any endomorphism field $A$, we set $(A \wedge \alpha)(v, w)=\alpha(w) A(v)-\alpha(v) A(w)$.

Proof of Proposition 2.10. Throughout this proof we will repeatedly use the formula for $\phi^{2}$ given in equation (2.4) without further notice.

We specialize equation (2.23) to $u=R_{\alpha}$ to get that $\nabla_{R_{\alpha}} \phi=0$. Thus using equation (2.14) we see that

$$
\begin{aligned}
\langle 2 h(v), w\rangle & =\left\langle\left[R_{\alpha}, \phi(v)\right]-\phi\left(\left[R_{\alpha}, v\right]\right), w\right\rangle=\left\langle\left(\nabla_{R_{\alpha}} \phi\right)(v)-\nabla_{\phi(v)} R_{\alpha}+\phi\left(\nabla_{v} R_{\alpha}\right), w\right\rangle \\
& =\left\langle\phi\left(\nabla_{v} R_{\alpha}\right)-\nabla_{\phi(v)} R_{\alpha}, w\right\rangle=\left\langle R_{\alpha}, \nabla_{\phi(v)} w+\nabla_{v} \phi(w)\right\rangle \\
& =\alpha\left(\nabla_{\phi(v)} w+\nabla_{v} \phi(w)\right) .
\end{aligned}
$$


Note that since $d \alpha(\phi(v), w)=-d \alpha(v, \phi(w))$, we have $\alpha([\phi(v), w])+\alpha([v, \phi(w)])=0$ and therefore

$$
\langle 2 h(v), w\rangle=\alpha\left(\nabla_{w} \phi(v)+\nabla_{\phi(w)} v\right)=\langle v, 2 h(w)\rangle .
$$

This proves equation (2.15).

For equation (2.16) we specialize equation (2.23) to $v=R_{\alpha}$ and obtain

$$
\begin{aligned}
\left\langle\left(\nabla_{u} \phi\right)\left(R_{\alpha}\right), w\right\rangle & =\frac{1}{2}\left\langle u^{\xi},\left(2 h-\theta^{\prime} I d\right) \wedge \alpha\left(R_{\alpha}, w\right)\right\rangle \\
& =-\left\langle u^{\xi}, h(w)\right\rangle+\theta^{\prime} / 2\left\langle u^{\xi}, w^{\xi}\right\rangle \\
& =-\langle h(u), w\rangle+\theta^{\prime} / 2\left\langle u^{\xi}, w^{\xi}\right\rangle
\end{aligned}
$$

where we then used the symmetry equation (2.15) of $h$ in the last equality. Since $\phi\left(R_{\alpha}\right)=0$, the left hand side of the above is $\left\langle\nabla_{u}\left(\phi\left(R_{\alpha}\right)\right)-\phi\left(\nabla_{u} R_{\alpha}\right), w\right\rangle=$ $-\left\langle\phi\left(\nabla_{u} R_{\alpha}\right), w\right\rangle$. Thus $\phi\left(\nabla_{u} R_{\alpha}\right)=h(u)-\left(\theta^{\prime} / 2\right) u^{\xi}$. Applying $\phi$, noting that $\nabla_{u} R_{\alpha}$ is tangent to $\xi$ and recalling that $\phi=J$ on $\xi$, we establish equation (2.16).

We now prove that $h$ anti-commutes with $\phi$; that is, we prove equation (2.17):

$$
\begin{aligned}
-\theta^{\prime}\langle\phi(v), u\rangle & +\langle u, h(\phi(v))+\phi(h(v))\rangle \\
& =\left\langle\frac{\theta^{\prime}}{2} \phi(u)-\phi(h(u)), v\right\rangle-\left\langle\frac{\theta^{\prime}}{2} \phi(v)-\phi(h(v)), u\right\rangle \\
& =\left\langle\nabla_{u} R_{\alpha}, v\right\rangle-\left\langle\nabla_{v} R_{\alpha}, u\right\rangle=-\left\langle R_{\alpha},[u, v]\right\rangle=d \alpha(u, v)=-\theta^{\prime}\langle u, \phi(v)\rangle .
\end{aligned}
$$

The second equality follows from equation (2.16), and the last equality follows from the fact that for all $u$ and $v$ in $T M$ we have

$$
g(u, \phi(v))=-\frac{1}{\theta^{\prime}} d \alpha(u, v),
$$

which in turn follows from equation (2.3) and the fact that $\phi(v) \in \xi$. Continuing we see $\langle u, h(\phi(v))+\phi(h(v))\rangle=0$ for all $u \in \xi$, thus establishing equation (2.17).

To prove equation (2.18) (which is the only one which appears to be new), we compute for any $v \in \xi$ (i.e. vector field extension of $v$ ),

$$
\begin{aligned}
I I(v, v)+I I(J v, J v) & =\left\langle\nabla_{v} v, R_{\alpha}\right\rangle+\left\langle\nabla_{J v} J v, R_{\alpha}\right\rangle=-\left\langle v, \nabla_{v} R_{\alpha}\right\rangle-\left\langle J v, \nabla_{J v} R_{\alpha}\right\rangle \\
& =\left\langle v,-\theta^{\prime} / 2 J v-h(J v)\right\rangle+\left\langle J v,-\theta^{\prime} / 2 J^{2} v-h\left(J^{2} v\right)\right\rangle \\
& =-\langle v, h(J v)\rangle+\langle J v, h(v)\rangle=-\langle v, h(J v)\rangle+\langle v, h(J v)\rangle=0,
\end{aligned}
$$

where the third equality follows from equation (2.16) and equation (2.17) has been used repeatedly.

We now prove equation (2.19). Let $v$ be a vector field tangent to $\xi$. We use that $J v=\phi(v)$ and $\nabla_{R_{\alpha}} \phi=0$ to get

$$
\nabla_{R_{\alpha}}(J v)=\nabla_{R_{\alpha}}(\phi(v))=\phi\left(\nabla_{R_{\alpha}} v\right) .
$$

In addition $\nabla_{R_{\alpha}} v$ is in $\xi$ by Proposition 2.6

Proof of Lemma 2.12. To establish equation (2.23) we notice that both sides of the equation are tensors in $u, v$ and $w$, so it suffices to establish the result when $u, v$ and $w$ are chosen to be elements of a basis for TM. We choose (local) vector fields $v_{1}, \ldots, v_{n}$ in $\xi$ such that $v_{1}, J v_{1}, \ldots, v_{n}, J v_{n}$ is an oriented orthonormal basis for $\xi$. In the computation below we assume that $u, v$ and $w$ are chosen from the set $\left\{v_{1}, J v_{1}, \ldots, v_{n}, J v_{n}, R_{\alpha}\right\}$. Notice that this implies that the lengths 
of $u, v, w, \phi(u), \phi(v)$ and $\phi(w)$ are constant as are their inner products with each other.

Equation (2.24) says that $\theta^{\prime} g(u, \phi(v))=-d \alpha(u, v)$ for any vectors $u$ and $v$. Using this and the fact that $d \alpha$ is closed we have

$$
\langle[u, v], \phi(w)\rangle+\langle[w, u], \phi(v)\rangle+\langle[v, w], \phi(u)\rangle=0 .
$$

Recall that the Koszul formula states that for any vector fields $u, v$ and $w$,

$2\left\langle\nabla_{u} v, w\right\rangle=u \cdot\langle v, w\rangle+v \cdot\langle u, w\rangle-w \cdot\langle u, v\rangle+\langle[u, v], w\rangle+\langle[w, u], v\rangle+\langle[w, v], u\rangle$.

Using this, equation (2.4) to compute $\phi^{2}$ and the previous equation we can begin our computation of $\nabla \phi$ as follows:

$$
\begin{aligned}
2\left\langle\left(\nabla_{u} \phi\right)(v), w\right\rangle= & 2\left\langle\nabla_{u}(\phi(v))-\phi\left(\nabla_{u} v\right), w\right\rangle \\
= & 2\left\langle\nabla_{u}(\phi(v)), w\right\rangle+2\left\langle\nabla_{u} v-\alpha\left(\nabla_{u} v\right) R_{\alpha}, \phi(w)\right\rangle \\
= & \langle[u, \phi(v)], w\rangle+\langle[w, u], \phi(v)\rangle+\langle[w, \phi(v)], u\rangle \\
& \quad+\langle[u, v], \phi(w)\rangle+\langle[\phi(w), u], v\rangle+\langle[\phi(w), v], u\rangle \\
= & \langle[u, \phi(v)], w\rangle+\langle[w, \phi(v)], u\rangle \\
& \quad+\langle[\phi(w), u], v\rangle+\langle[\phi(w), v], u\rangle-\langle[v, w], \phi(u)\rangle .
\end{aligned}
$$

Substituting $\phi(v)$ for $v$ and $\phi(w)$ for $w$ in equation (2.25) and using equation (2.4) to compute $\phi^{2}$ we learn that

$$
\begin{aligned}
-\langle[u, \phi(v)], w\rangle & +\alpha(w)\left\langle[u, \phi(v)], R_{\alpha}\right\rangle-\langle[\phi(w), u], v\rangle \\
& +\alpha(v)\left\langle[\phi(w), u], R_{\alpha}\right\rangle+\langle[\phi(v), \phi(w)], \phi(u)\rangle=0 .
\end{aligned}
$$

This may be used to eliminate the first and third terms in the preceding equation, which becomes

$$
\begin{gathered}
2\left\langle\left(\nabla_{u} \phi\right)(v), w\right\rangle=\langle[w, \phi(v)], u\rangle+\langle[\phi(w), v], u\rangle-\langle[v, w], \phi(u)\rangle+\alpha(w)\left\langle[u, \phi(v)], R_{\alpha}\right\rangle \\
+\alpha(v)\left\langle[\phi(w), u], R_{\alpha}\right\rangle+\langle[\phi(v), \phi(w)], \phi(u)\rangle .
\end{gathered}
$$

Note that

$$
\begin{aligned}
\left\langle[u, \phi(v)], R_{\alpha}\right\rangle & =\alpha([u, \phi(v)])=-d \alpha(u, \phi(v)) \\
& =-d \alpha\left(u^{\xi}, J v^{\xi}\right)=-\theta^{\prime}\left\langle u^{\xi}, v^{\xi}\right\rangle .
\end{aligned}
$$

Using this and the analogous formula with $w$ instead of $v$ allows us to rewrite equation (2.26) as

$$
\begin{aligned}
2\left\langle\left(\nabla_{u} \phi\right)(v), w\right\rangle= & \langle[w, \phi(v)], u\rangle+\langle[\phi(w), v], u\rangle-\langle[v, w], \phi(u)\rangle+\langle[\phi(v), \phi(w)], \phi(u)\rangle \\
& -\theta^{\prime}\left\langle u^{\xi},(I d \wedge \alpha)(v, w)\right\rangle .
\end{aligned}
$$

We want to rewrite the first line of this equation in terms of $[J, J]$. In the following computation we use the definition of $[J, J]$, the fact that, for any $u$, $u^{\xi}=u-\alpha(u) R_{\alpha}, \phi(u)=J u^{\xi}$ and the fact that $\left[J v^{\xi}, J w^{\xi}\right]-\left[v^{\xi}, w^{\xi}\right]$ is tangent to $\xi$ for any vector fields $v$ and $w$ :

$$
\begin{aligned}
{[J, J]\left(v^{\xi}, J w^{\xi}\right)=} & -\left[v^{\xi}, J w^{\xi}\right]-\left[J v^{\xi}, w^{\xi}\right]-J\left(\left[J v^{\xi}, J w^{\xi}\right]-\left[v^{\xi}, w^{\xi}\right]\right) \\
= & -\left[v-\alpha(v) R_{\alpha}, \phi(w)\right]-\left[\phi(v), w-\alpha(w) R_{\alpha}\right] \\
& -\phi\left([\phi(v), \phi(w)]-\left[v-\alpha(v) R_{\alpha}, w-\alpha(w) R_{\alpha}\right]\right) .
\end{aligned}
$$


Next we use that $\alpha(v)$ and $\alpha(w)$ are constant to get them out of Lie brackets and we use equation (2.14) to get

$[J, J]\left(v^{\xi}, J w^{\xi}\right)=-[v, \phi(w)]-[\phi(v), w]-\phi([\phi(v), \phi(w)])+\phi([v, w])-2(h \wedge \alpha)(v, w)$.

Comparing the above with equation (2.28) and noticing that both $[J, J]$ and $h \wedge \alpha$ take values in $\xi$ gives the announced equation (2.23).

Proof of Proposition 2.11. Because $h$ restricted to $\xi$ is symmetric with respect to $g$, it is diagonalizable in an orthonormal basis. Assume that we have a local vector field $v$ with $h(v)=\lambda v$, for some function $\lambda$. Since $h$ anti-commutes with $J$ we see that $h(J v)=-\lambda J v$. So locally we can get an orthonormal frame $\left\{v_{1}, J v_{1}, \ldots, v_{n}, J v_{n}\right\}$ for $\xi$ such that $h v_{i}=\lambda_{i} v_{i}$ and $h J v_{i}=-\lambda_{i} J v_{i}$ for some non-negative functions $\lambda_{i}$.

Let $v=v_{i}$ and $\lambda=\lambda_{i}$ for some $1 \leq i \leq n$. From equation (2.16) we have

$$
\begin{gathered}
\nabla_{v} R_{\alpha}=\left(\theta^{\prime} / 2-\lambda\right) J v, \\
\nabla_{J v} R_{\alpha}=-\left(\theta^{\prime} / 2+\lambda\right) v .
\end{gathered}
$$

So, for any vector $u$ in $\xi$ we can write $u=\sum\left(a_{i} v_{i}+b_{i} J v_{i}\right)$, and the above equation gives

$$
\left\|\nabla_{u} R_{\alpha}\right\|^{2}=\sum\left(\left(\frac{\theta^{\prime}}{2}+\lambda_{i}\right)^{2} b_{i}^{2}+\left(\frac{\theta^{\prime}}{2}-\lambda_{i}\right)^{2} a_{i}^{2}\right) .
$$

Using also equation (2.5) claiming $\nabla_{R_{\alpha}} R_{\alpha}=0$ and the fact that each $\lambda_{i}$ is nonnegative and $\theta^{\prime}$ is positive, we get $\left\|\nabla R_{\alpha}\right\|=\theta^{\prime} / 2+\max _{i} \lambda_{i}$.

Let $Q$ be the quadratic form on $\xi$ defined by

$$
Q(u)=\left\langle\operatorname{Rm}\left(u, R_{\alpha}\right) R_{\alpha}, u\right\rangle+\left\langle\operatorname{Rm}\left(J u, R_{\alpha}\right) R_{\alpha}, J u\right\rangle,
$$

where $\mathrm{Rm}$ is the Riemann curvature tensor defined by $\operatorname{Rm}(X, Y) Z=\nabla_{X}\left(\nabla_{Y} Z\right)-$ $\nabla_{Y}\left(\nabla_{X} Z\right)-\nabla_{[X, Y]} Z$ so both terms are sectional curvatures if $\|u\|=1$. Let $u$ be a vector field tangent to $\xi$. Because of Proposition 2.6 and the symmetry of the Levi-Civita connexion, $\left[R_{\alpha}, u\right]$ is also tangent to $\xi$. In the following computation we get rid of Lie brackets using that the Levi-Civita connexion is torsion free and transform all terms of type $\nabla_{v} R_{\alpha}$ using Proposition 2.6 and equation (2.16). We also use that $h$ and $J$ anti-commute (equation (2.17) ) and equation (2.19):

$$
\begin{aligned}
\left\langle\operatorname{Rm}\left(u, R_{\alpha}\right) R_{\alpha}, u\right\rangle= & \left\langle\nabla_{\left[R_{\alpha}, u\right]} R_{\alpha}, u\right\rangle-\left\langle\nabla_{R_{\alpha}}\left(\nabla_{u} R_{\alpha}\right), u\right\rangle+\left\langle\nabla_{u}\left(\nabla_{R_{\alpha}} R_{\alpha}\right), u\right\rangle \\
= & \left\langle J\left(\theta^{\prime} / 2-h\right)\left[R_{\alpha}, u\right], u\right\rangle-\left\langle\nabla_{R_{\alpha}}\left(J\left(\theta^{\prime} / 2-h\right) u\right), u\right\rangle \\
= & \left\langle J\left(\theta^{\prime} / 2-h\right) \nabla_{R_{\alpha}} u+\left(\left(\theta^{\prime} / 2\right)^{2}-h^{2}\right) u, u\right\rangle \\
& -\left\langle\nabla_{R_{\alpha}}\left(J\left(\theta^{\prime} / 2-h\right) u\right), u\right\rangle \\
= & \left\langle\left(\left(\theta^{\prime} / 2\right)^{2}-h^{2}\right) u-\left(\nabla_{R_{\alpha}} h\right) J u, u\right\rangle .
\end{aligned}
$$

Adding the same formula applied to $J u$ leads to

$$
Q(u)=2\left\langle\left(\left(\theta^{\prime} / 2\right)^{2}-h^{2}\right) u, u\right\rangle .
$$

So $Q$ is a quadratic form on $\xi$ having eigenvalues (with respect to the inner product g) $\mu_{i}=2\left(\left(\theta^{\prime} / 2\right)^{2}-\lambda_{i}^{2}\right)$. The minimum of $Q$ on the unit sphere is $\min \left(\mu_{i}\right)=$ $2\left(\theta^{\prime} / 2\right)^{2}-2 \max \left(\lambda_{i}\right)^{2}$.

So we have proved

$$
\max \lambda_{i}=\sqrt{\left(\frac{\theta^{\prime}}{2}\right)^{2}-\frac{1}{2} \min _{u \in \xi,\|u\|=1} Q(u)}
$$


(in particular, the term under the square root is non-negative). This gives the formula we wanted for $\left\|\nabla R_{\alpha}\right\|$.

Regarding equation (2.22), we note that equation (2.23) gives

$$
\left|\left\langle\left(\nabla_{u} \phi\right)(v), w\right\rangle\right| \leq \frac{1}{2}\left(\|[J, J]\|+2\left\|2 h-\theta^{\prime} I d\right\|\right)\|u\| \cdot\|v\| \cdot\|w\|,
$$

and clearly $\left\|2 h-\theta^{\prime} I d\right\|=2 \max \lambda_{i}+\theta^{\prime}$, so we have the announced bound.

\section{A tightness Radius estimate}

This section is devoted to the proof of Theorem 1.1. It uses standard holomorphic curve arguments and a key comparison of Riemannian and almost complex convexity in symplectizations of contact metric manifolds. This comparison is explained in Subsection 3.2 after we recall a few results about Riemannian convexity in Subsection 3.1. We then recall the definition of PS-overtwisted manifolds and their relevant properties in Subsection 3.3 before proving the theorem in Subsection 3.4.

3.1. Convexity in Riemannian geometry. Let $S$ be a cooriented hypersurface in a Riemannian manifold $\left(M^{n}, g\right)$. We say that $S$ is geodesically convex at $p$ if its second fundamental form is positive definite at $p$. This has to do with convexity because it forces geodesics which are tangent to $S$ at $p$ to stay (locally) on one side of $S$. Let $f$ be a function defined on a small neighborhood $U$ of $p$ such that $S \cap U$ is a regular level set of $f$ and $\nabla f$ defines the coorientation of $S$. Then $S$ is geodesically convex at $p$ if and only if the Hessian of $f$ at $p$ is positive definite.

The hypersurfaces we will consider are geodesic spheres, so the relevant functions are distances from points. To any real number $k$, one associates the reference function

$$
\operatorname{ct}_{k}(r)= \begin{cases}\sqrt{k} \cot (\sqrt{k} r), & \text { if } k>0, \\ \frac{1}{r}, & \text { if } k=0, \\ \sqrt{-k} \operatorname{coth}(\sqrt{-k} r), & \text { if } k<0 .\end{cases}
$$

Proposition 3.1 (See e.g. [17, Theorem 27, p. 175]). Let $\left(M^{n}, g\right)$ be a Riemannian manifold with $\sec (g) \leq K$ for some real number $K$. Let $r$ be any radius below the injectivity radius $\operatorname{inj}(g)$ and $p$ any point in $M$. If $K$ is non-positive, then the Hessian of the distance function

$$
\mathrm{r}_{p}: M^{n} \rightarrow \mathbb{R}: q \mapsto d(p, q)
$$

is positive definite on the ball of radius $r$ about $p, B_{p}(r)$. If $K$ is positive, then the same holds provided $r$ is less than $\frac{\pi}{2 \sqrt{K}}$.

More generally, the Hessian of $r$ satisfies

$$
\nabla^{2} \mathrm{r}_{p} \geq \operatorname{ct}_{K}(r) g
$$

and $\operatorname{ct}_{K}(r)$ is positive whenever $K$ is non-positive or $K$ is positive and $r<\frac{\pi}{2 \sqrt{K}}$.

The convexity radius $\operatorname{conv}(g)$ of Theorem 1.1 refers to the maximum $r>0$ such that all geodesic balls of radius less than or equal to $r$ have geodesically convex boundary as hypersurfaces. 
3.2. Pseudo-convexity in symplectizations. We consider the setup analogous to the one in $[8$ but in dimension $\geq 3$. The symplectization of a contact manifold $(M, \xi)$ equipped with a distinguished contact form $\alpha$ is the product $W:=\mathbb{R} \times M$, equipped with the symplectic form $\omega=d\left(e^{t} \alpha\right)$, where $t$ is the coordinate on $\mathbb{R}$. Let $J$ be a complex structure on $\xi$ that is compatible with $\left.(d \alpha)\right|_{\xi}$ and extended to $T W$ by setting $J \partial_{t}=R_{\alpha}$.

Let $U$ be a regular sublevel set of some function $f: M \rightarrow \mathbb{R}$ and $S=\partial U$. We can think of $f$ as a function on $W$ (by composing with the projection $W \rightarrow M$ ), and thus we get the regular sublevel set $\Omega=\mathbb{R} \times U$ with boundary $\Sigma=\mathbb{R} \times S$. The complex tangencies to $\Sigma$,

$$
\mathcal{C}_{\Sigma}=T \Sigma \cap J(T \Sigma)
$$

can be described as the kernel of the 1 -form $d f \circ J$. The form

$$
L(u, v)=-d(d f \circ J)(u, J v)
$$

is called the Levi form of $\Sigma$. Recall that $\Sigma$ is said to be pseudoconvex, or strictly pseudoconvex, if $L(v, v) \geq 0$, respectively $L(v, v)>0$, for all non-zero $v \in \mathcal{C}_{\Sigma}$.

We also extend the metric $g$ on $M$ to $W$ by $g+d t \otimes d t$. The following result which relates Riemannian and symplectic convexity should be compared with the analogous result in Kähler geometry [11, Lemma, p. 646] and in dimension 3 [8].

Proposition 3.2. Let $(\alpha, g, J)$ be a compatible metric structure on the contact manifold $\left(M^{2 n+1}, \xi\right)$. Then, using the notation above, for any $v \in \mathcal{C}_{\Sigma}$ we have

$$
L(v, v)=\nabla^{2} f(v, v)+\nabla^{2} f(J v, J v) .
$$

The rest of this subsection is devoted to the proof of the preceding proposition and can be safely skipped on first reading. For convenience of notation we denote $R_{\alpha}$ by $n$ in the computations. We define two bundle maps $A: \xi \rightarrow T W$ and $B: \xi \rightarrow T W$ by

and

$$
A(v)=J[J v, v]-\nabla_{v} v-\nabla_{J v} J v
$$

$$
B(v)=J[v, n]+\nabla_{J v} n+\nabla_{n} J v .
$$

One can easily check that the values $A$ and $B$ at a point only depend on the vector at the point and not on the local extension to local vector fields (more precisely $B$ is a tensor and $A$ is a quadratic bundle map). We begin with the following observation (which originally appeared for metric contact 3-manifolds in [8]).

Lemma 3.3. Under the hypotheses of Proposition 3.2, we have

$$
\begin{aligned}
& -L(v, v)+\nabla^{2} f(v, v)+\nabla^{2} f(J v, J v) \\
& \quad=d f\left(A\left(v^{\xi}\right)+a B\left(J v^{\xi}\right)-b B\left(v^{\xi}\right)-\left(a^{2}+b^{2}\right) \nabla_{n} n\right),
\end{aligned}
$$

where the vector $v \in \mathcal{C}_{\Sigma}$ is written as $v=v^{\xi}+a n+b \partial_{t}$, with $v^{\xi} \in \xi$.

Proof. We extend $v$ to a vector field on $W$ as $v=v^{\xi}+a n+b \partial_{t}$, with $v^{\xi} \in \xi$ invariant under translation in the $\mathbb{R}$ direction, and $a$ and $b$ are constants. We first compute

$$
\begin{aligned}
\nabla^{2} f(v, v)+\nabla^{2} f(J v, J v) & =v \cdot(d f(v))-\left(\nabla_{v} v\right) \cdot f+(J v) \cdot(d f(J v))-\left(\nabla_{J v} J v\right) \cdot f \\
& =v \cdot(d f(v))+(J v) \cdot(d f(J v))-d f\left(\nabla_{v} v+\nabla_{J v} J v\right) .
\end{aligned}
$$

And, using the formula $d \alpha(u, w)=u \cdot \alpha(w)-w \cdot \alpha(u)-\alpha([u, w])$ we have

$$
d(d f \circ J)(v, J v)=-v \cdot(d f(v))-(J v) \cdot(d f(J v))+d f(J[J v, v]) .
$$


Adding the two preceding equations, we obtain

$$
-L(v, v)+\nabla^{2} f(v, v)+\nabla^{2} f(J v, J v)=d f\left(J[J v, v]-\nabla_{v} v-\nabla_{J v} J v\right) .
$$

Decomposing $v$ as $v^{\xi}+a n+b \partial_{t}$ as in the statement of the lemma and using $\nabla_{\partial_{t}} v=0$ we compute

$$
\begin{aligned}
J[J v, v] & =J\left[J v^{\xi}, v^{\xi}\right]+a J\left[J v^{\xi}, n\right]+b J\left[n, v^{\xi}\right], \\
\nabla_{v} v & =\nabla_{v^{\xi}} v^{\xi}+a\left(\nabla_{v^{\xi}} n+\nabla_{n} v^{\xi}\right)+a^{2} \nabla_{n} n, \\
\nabla_{J v} J v & =\nabla_{J v^{\xi}} J v^{\xi}+b\left(\nabla_{J v^{\xi}} n+\nabla_{n} J v^{\xi}\right)+b^{2} \nabla_{n} n .
\end{aligned}
$$

Thus we see that $-L(v, v)+\nabla^{2} f(v, v)+\nabla^{2} f(J v, J v)$ equals

$$
d f\left(A\left(v^{\xi}\right)+a B\left(J v^{\xi}\right)-b B\left(v^{\xi}\right)-\left(a^{2}+b^{2}\right) \nabla_{n} n\right),
$$

giving the announced formula.

Now we establish Proposition 3.2 . We begin by computing an expression for the operators $A$ and $B$ from Lemma 3.3 .

Lemma 3.4. Under the hypotheses of Proposition [3.2, for any vector $v$ in $\xi$ we have

$$
A(v)=-\theta^{\prime}\|v\|^{2} \partial_{t} \quad \text { and } \quad B(v)=-\theta^{\prime} v .
$$

Proof. We can use equations (2.16), (2.17), and (2.19) to compute $B(v)$ :

$$
\begin{aligned}
B(v) & =J[v, n]+\nabla_{J v} n+\nabla_{n} J v=J \nabla_{v} n-J \nabla_{n} v+\nabla_{J v} n+\nabla_{n} J v \\
& =J \nabla_{v} n+\nabla_{J v} n=\left(-\theta^{\prime} / 2 v+h(v)\right)+J\left(\theta^{\prime} / 2 J v-h(J v)\right)=-\theta^{\prime} v .
\end{aligned}
$$

We now compute $A(v)$ by projecting it to $n, \partial_{t}$ and $\xi$. Starting with the projection to $n$ we use equation (2.18) to conclude that

$$
\begin{aligned}
\langle A(v), n\rangle & =\langle J[J v, v], n\rangle-\left\langle\nabla_{v} v, n\right\rangle-\left\langle\nabla_{J v} J v, n\right\rangle \\
& =\left\langle[J v, v], \partial_{t}\right\rangle-I I(v, v)-I I(J v, J v)=0 .
\end{aligned}
$$

Continuing with the projection to $\partial_{t}$ we have

$$
\begin{aligned}
\left\langle A(v), \partial_{t}\right\rangle & =\left\langle J[J v, v], \partial_{t}\right\rangle=-\langle[J v, v], n\rangle \\
& =-\left\langle\nabla_{J v} v, n\right\rangle+\left\langle\nabla_{v} J v, n\right\rangle=\left\langle v, \nabla_{J v} n+J \nabla_{v} n\right\rangle .
\end{aligned}
$$

From the last line of our computations of $B(v)$ above we conclude that $\left\langle A(v), \partial_{t}\right\rangle=$ $-\theta^{\prime}\|v\|^{2}$. Finally, we can compute, for any $w$ in $\xi$,

$$
\begin{aligned}
\langle A(v), w\rangle & =\left\langle J[J v, v]-\nabla_{v} v-\nabla_{J v} J v, w\right\rangle=\left\langle\phi([\phi(v), v])-\nabla_{v} v-\nabla_{\phi(v)} \phi(v), w\right\rangle \\
& =\left\langle\phi\left(\nabla_{\phi(v)} v\right)-\phi\left(\nabla_{v} \phi(v)\right)-\nabla_{v} v-\nabla_{\phi(v)} \phi(v), w\right\rangle \\
& =-\left\langle\left(\nabla_{\phi(v)} \phi\right)(v), w\right\rangle+\left\langle\left(\nabla_{v} \phi\right)(\phi(v)), w\right\rangle \\
& =-\frac{1}{2}\langle[J, J](v, J w), J v\rangle+\frac{1}{2}\langle[J, J](J v, J w), v\rangle \quad \text { by equation (2.23) } \\
& =\frac{1}{2}\langle J[J, J](v, J w)+[J, J](J v, J w), v\rangle .
\end{aligned}
$$

Using the definition of $[J, J]$ and the fact that $J^{2}=-i d$, one can check that, for any $v$ and $w$ in $\xi, J[J, J](v, J w)+[J, J](J v, J w)=0$. Hence $A(v)$ has no component in $\xi$. 
Proof of Proposition 3.2. Combining equation (2.5) and Lemmas 3.3 and 3.4 we see that

$$
\begin{aligned}
-L(v, v)+\nabla^{2} f(v, v)+\nabla^{2} f(J v, J v) & =d f\left(-\theta^{\prime}\left(\left\|v^{\xi}\right\|^{2} \partial_{t}+a J v_{\xi}-b v_{\xi}\right)\right) \\
& =-\theta^{\prime} d f\left(\|v\|^{2} \partial_{t}+a J v-b v\right)=0,
\end{aligned}
$$

where the last equality follows since $v \in \mathcal{C}_{\Sigma}$.

3.3. Bordered Legendrian open books. Let $N$ be a compact manifold with non-empty boundary. A relative open book on $N$ is a pair $(B, \theta)$ where

- the binding $B$ is a non-empty codimension 2 submanifold in the interior of $N$ with trivial normal bundle, and

- $\theta: N \backslash B \rightarrow S^{1}$ is a fibration whose fibers are transverse to $\partial N$ and which coincides in a neighborhood $B \times D^{2}$ of $B=B \times\{0\}$ with the normal angular coordinate.

Definition 3.5 (Massot, Niederkrüger and Wendl [14]). Let $(M, \xi)$ be a $(2 n+1)$ dimensional contact manifold. A compact $(n+1)$-dimensional submanifold $N \hookrightarrow M$ with boundary is called a bordered Legendrian open book (abbreviated bLob) if it has a relative open book $(B, \theta)$ such that

(i) all fibers of $\theta$ are Legendrian, and

(ii) the boundary of $N$ is Legendrian.

If such a submanifold exists, then $(M, \xi)$ is called PS-overtwisted.

We notice that the notion of a plastikstufe defined in 15 is a special case of a bLob where the fibers of the bLob are of the form $B \times[0,1]$. The term PS-overtwisted originally referred to the existence of a plastikstufe in a contact manifold, but it was generalized in 14. Although it is not certain if this definition is a definitive generalization of overtwisted to higher dimensional manifolds since it may or may not be equivalent to the definition in [4, it does have some of the properties of 3-dimensional overtwisted contact manifolds. In particular, we have the following result.

Theorem 3.6 (Niederkrüger [14,15]). If $(M, \xi)$ is a PS-overtwisted contact manifold, then it cannot be symplectically filled by a semi-positive symplectic manifold. If the dimension of $M$ is less than 7, then it cannot be filled by any symplectic manifold.

A $2 n$-dimensional symplectic manifold $(X, \omega)$ is called semi-positive if every element $A \in \pi_{2}(X)$ with $\omega(A)>0$ and $c_{1}(A) \geq 3-n$ satisfies $c_{1}(A)>0$. Note that all Stein and exact symplectic manifolds are semi-positive.

The presence of a bLob also has dynamical consequences, and they will be crucial in our proof of Theorem 1.1. Recall that the Weinstein conjecture asserts that any Reeb vector field on a closed contact manifold has some closed Reeb orbits. Contractible Reeb orbits do not always exist, but the considerations in [14 allows us to slightly generalize the main theorem in 1 resulting in the following theorem.

Theorem 3.7 (Albers and Hofer [1]). Let $(M, \xi)$ be a closed PS-overtwisted contact manifold. Then every Reeb vector field associated to $\xi$ has a contractible periodic orbit. 
3.4. Proof of the tightness radius estimate. We can now prove Theorem 1.1 , which claims that a ball $B(x, r)$ whose radius $r$ is below the convexity radius in a contact metric manifold cannot contain a bLob.

Proof of Theorem 1.1. If we assume the existence of a bLob, then one can start a family of holomorphic disks as in [15. Because of the Levi form computation of Proposition 3.2, the boundary of a convex ball lifts to a pseudo-convex hypersurface in the symplectization. The weak maximum principle for elliptic operators then guarantees that holomorphic curves cannot "touch from the inside" this hypersurface. This allows us to use the strategy of [1] without any modification and prove the existence of a closed Reeb orbit $\gamma$ inside the ball $B$.

However, such an orbit would be a closed geodesic according to Proposition 2.6. Those cannot exist inside $B$ because it would have to be somewhere tangent to a sphere $S\left(x, r_{0}\right)$, for some $r_{0}$, with $\gamma$ lying inside the ball $B\left(x, r_{0}\right)$. Of course $r_{0}$ is also below the convexity radius, so $\partial B\left(x, r_{0}\right)$ cannot be "touched from the inside" by a geodesic, and we get a contradiction.

The part of Theorem 1.1 relating to curvature follows from the above and the estimate on the Hessian of the radial function given in Proposition 3.1 .

Proof of Corollary 1.2. Note that pull-backs of $\xi$ and the metric to any covering space are compatible and the sectional curvature is non-positive. It is well known, by Hadamard Theorem [5. Theorem IV.1.3, p. 192], that the universal cover of a manifold with non-positive curvature is $\mathbb{R}^{2 n+1}$ and the space is exhausted by geodesic balls. Moreover, the convexity radius of the universal cover is infinite. Thus Theorem 1.1 says that a ball of any radius is bLob free. The result follows.

\section{A quantitative Darboux theorem in any Dimension}

In this section, we establish an estimate on the Darboux radius of a contact manifold with a compatible metric structure. We begin by introducing a number of quantities used throughout this section that depend on the dimension, the instantaneous rotation $\theta^{\prime}$ of the contact structure and bounds on curvature and injectivity radius. Unless said otherwise, we assume that the sectional curvature of $g$ is between $\kappa$ and $K$ :

$$
\kappa \leq \sec (P) \leq K,
$$

for any 2-plane $P$ in $T M$. Further, we define

$$
r_{\text {max }}= \begin{cases}\min \left(\operatorname{inj}(g), \frac{\pi}{2 \sqrt{K}}\right), & \text { if } K>0, \\ \operatorname{inj}(g), & \text { if } K \leq 0 .\end{cases}
$$

We also define the quantities

$$
\begin{aligned}
& A=\frac{4}{3} \sqrt{2 n-1}|\sec (g)| \text { and } \\
& B=\frac{\theta^{\prime}}{2}+\sqrt{\frac{\left(\theta^{\prime}\right)^{2}}{4}-\frac{1}{2} \min _{u \in \xi,\|u\|=1}\left(K\left(u, R_{\alpha}\right)+K\left(J u, R_{\alpha}\right)\right)}
\end{aligned}
$$

where $|\sec (g)|$ is the maximum in absolute value sectional curvature over $(M, g)$, and $K(u, v)$ denotes the sectional curvature of the plane spanned by $u$ and $v$. The square root appearing in $B$ is well defined thanks to Proposition 2.11. (Note 
that $A$ and $B$ have nothing to do with the operators appearing in the Levi form computation of the preceding section.)

In addition to the reference function $\mathrm{ct}_{k}$ defined by equation (3.1), we will need functions $\mathrm{sn}_{k}$, also indexed by a real number $k$ :

$$
\operatorname{sn}_{k}(r)= \begin{cases}\frac{1}{\sqrt{k}} \sin (\sqrt{k} r), & \text { if } k>0, \\ r, & \text { if } k=0, \\ \frac{1}{\sqrt{-k}} \sinh (\sqrt{-k} r), & \text { if } k<0 .\end{cases}
$$

These functions combine with $A$ and $B$ and an upper bound $K$ on the sectional curvature to define

$$
Q(r):=\operatorname{sn}_{K}^{-1}\left(\left(1-B r-\frac{1}{2} A r^{2}\right) \operatorname{sn}_{K}(r)\right)
$$

and the constants

$$
\begin{aligned}
\bar{H}_{1} & = \begin{cases}\sqrt{2}, & \text { if } \kappa \geq 0, \\
\sqrt{1+\left(\frac{\mathrm{sn}_{\kappa}\left(r_{\max }\right)}{r_{\max }}\right)^{2},} & \text { if } \kappa<0,\end{cases} \\
\bar{H}_{2} & =\frac{8}{3}|\sec (g)| \bar{H}_{1} r_{\max }, \text { and } \\
\bar{H} & =2 \bar{H}_{1} \bar{H}_{2}+(\|[J, J]\| / 2+2 B) \bar{H}_{1}^{2} .
\end{aligned}
$$

Combining all these numbers, and using the fact that $B$ is positive, we define

$$
r_{\tau}=\min \left(\frac{\operatorname{inj}(g)}{2}, \frac{\pi}{2 \sqrt{K}}, \frac{2}{\sqrt{2 A+B^{2}}+B}, \frac{1}{(1+2 n(n-1)) \bar{H}}\right) .
$$

We are now ready to state the refined version of Theorem 1.5, with a refined estimate, which will be proven in this section.

Theorem 4.1. Given a compatible metric structure $(\alpha, g, J)$ on $(M, \xi)$, with sectional curvature bounded as in (4.1), the Darboux radius admits the following bound:

$$
\delta(M, \xi) \geq Q\left(r_{\tau}\right) .
$$

First we explain how the coarser version announced in the introduction follows from the above result.

Proof of Theorem 1.5. We now discuss how this bound simplifies if we are willing to assume that the sectional curvature of $g$ is in $[-K, K]$ for some positive constant $K$ and we roughly estimate the complicated functions appearing above. We set

$$
\rho=\max \left(\theta^{\prime}, \sqrt{K},\|[J, J]\|\right) .
$$

In particular, $A \leq 4 \sqrt{2 n-1} \rho^{2} / 3 \leq 2 \sqrt{n} \rho^{2}$. Using that $\sqrt{\left(1+x^{2}\right)} \leq 1+x$ for non-negative $x$ we estimate

$$
\begin{aligned}
B & \leq \frac{\theta^{\prime}}{2}\left(1+\sqrt{1+\frac{4 K}{\theta^{\prime 2}}}\right) \leq \frac{\theta^{\prime}}{2}\left(1+1+\frac{2 \sqrt{K}}{\theta^{\prime}}\right) \\
& \leq 2 \rho .
\end{aligned}
$$


Also, we compute

$$
\begin{aligned}
\bar{H}_{1} & =\sqrt{1+\left(\frac{\mathrm{sn}-K\left(r_{\max }\right)}{r_{\max }}\right)^{2}}=\sqrt{1+\left(\frac{\sinh \left(\sqrt{K} r_{\max }\right)}{\sqrt{K} r_{\max }}\right)^{2}} \\
& \leq \sqrt{1+\left(\frac{2}{\pi} \sinh \left(\frac{\pi}{2}\right)\right)^{2}}<2
\end{aligned}
$$

because $\sqrt{K} r_{\max }$ is always less than $\pi / 2$ (there are two cases to check depending on what term attains the min in the definition of $r_{\max }$ ). So $\bar{H}_{1} \leq 2$ and $\bar{H}_{2} \leq \frac{8 \pi \rho}{3}$ and $\bar{H} \leq 52 \rho$.

On the other hand, our estimates on $A$ and $B$ give

$$
\sqrt{2 A+B^{2}}+B \leq 6 n^{1 / 4} \rho .
$$

So, the terms appearing in the definition of $r_{\tau}$ are estimated as follows:

$$
\frac{2}{\sqrt{2 A+B^{2}}+B} \geq \frac{1}{3 n^{1 / 4} \rho} \quad \text { and } \quad \frac{1}{(1+2 n(n-1)) \bar{H}} \geq \frac{1}{104 n^{2} \rho} .
$$

Note that the second bound above is always less than the first one, so, setting $d_{n}:=1 /\left(104 n^{2}\right)$, we see that

$$
r_{\tau} \geq \min \left(\operatorname{inj}(g), \frac{d_{n}}{\rho}\right) .
$$

It remains to estimate $Q\left(r_{0}\right)$ for $r_{0} \leq d_{n} / \rho$. Our estimates on $A$ and $B$ give

Therefore

$$
1-B r_{0}-\frac{1}{2} A r_{0}^{2} \geq 1-2 d_{n}-d_{n}^{2} \sqrt{n} \geq \frac{97}{100} \text {. }
$$

$$
Q\left(r_{0}\right) \geq \frac{1}{\sqrt{K}} \arcsin \left(\frac{97}{100} \sin \left(\sqrt{K} r_{0}\right)\right) \geq \frac{1}{\sqrt{K}} \frac{1}{2} \sqrt{K} r_{0}=\frac{r_{0}}{2},
$$

which yields the promised Darboux radius estimate.

We will now prove Theorem 4.1 modulo a number of propositions which will be proved in subsequent subsections. The goal is to embed a large geodesic ball in our contact manifold into the standard contact $\mathbb{R}^{2 n+1}$. The latter is the contactization of the standard Liouville structure on $\mathbb{R}^{2 n}$ and we will compare it to some contactization of a natural exact symplectic manifold inside our given contact metric manifold $M$. Recall a Liouville manifold is a pair $(W, \lambda)$ where $d \lambda$ is a symplectic form on $W$ and $\lambda$ restricted to the boundary of $W$ is a contact form for a positive contact structure. Also recall that the contactization of an exact symplectic manifold $(W, \beta)$, and in particular a Liouville manifold, is $\mathbb{R} \times W$ equipped with the contact structure $\operatorname{ker}(d t+\beta)$.

Given any point $p$ in $M$ and the contact hyperplane $\xi_{p}$ at $p$, the geodesic disk $\mathrm{D}(r)$ centered at $p$ of radius $r$ and tangent to $\xi_{p}$ is given as the image of the restriction of the exponential map to the disk of radius $r$ in $\xi_{p}$, that is,

$$
\mathrm{D}(r)=\exp _{p}\left(D_{\xi}(r)\right)
$$

where $D_{\xi}(r)=\left(\left\{v \in \xi_{p} ;|v|<r\right\}\right)$. Denoting the Reeb flow by $\Phi(t, \mathbf{x}): \mathbb{R} \times M \longrightarrow$ $M$ we define the map

$$
E: \mathbb{R} \times D_{\xi}(r) \rightarrow M:(t, v) \mapsto \Phi\left(t, \exp _{p}(v)\right)
$$


and the $R_{\alpha}$-invariant "cylindrical" neighborhood $\mathrm{C}(r)$ of $\mathrm{D}(r)$ to be the image of $E$. Of course $\mathrm{C}(r)$ is not, in general, an embedded submanifold of $M$, but for $r$ small enough $\mathrm{D}(r)$ will be an embedded disk and $R_{\alpha}$ will be transverse to $\mathrm{D}(r)$. For such an $r, \mathrm{C}(r)$ will then contain embedded neighborhoods of $\mathrm{D}(r)$, for example $E\left((-\epsilon, \epsilon) \times D_{\xi}(r)\right)$, for sufficiently small $\epsilon$. To prove Theorem 4.1 we will proceed in the following steps.

Step I. Find an estimate on the radius $r$ so that $R_{\alpha}$ is transverse to $\mathrm{D}(r)$.

Step II. Find an estimate on the radius $r$ so that the pull-back of the contact structure $\xi$ to $\mathbb{R} \times D_{\xi}(r)$ via $E$ embeds into the standard contact $\mathbb{R}^{2 n+1}$.

Step III. Find an estimate on the size of a geodesic ball about $p$ that embeds in $M$ and is contained in $\mathrm{C}(r)$.

We will first list several propositions that will be proven in the following subsections that give the estimates indicated in the outline above and then assemble them into a proof of Theorem 4.1. The estimate in Step I is given in the following proposition.

Proposition 4.2 (proved in Section 4.1). Given a compatible metric structure $(\alpha, g, J)$ on the contact manifold $(M, \xi)$, the disk $\mathrm{D}\left(r_{0}\right)$ is embedded and the Reeb vector field $R_{\alpha}$ is positively transverse to it if

$$
r_{0}<r_{\text {内 }}:=\min \left\{\operatorname{inj}(g), \frac{\pi}{2 \sqrt{K}}, \frac{2}{\sqrt{2 A+B^{2}}+B}\right\},
$$

where the constants are defined at the beginning of this section. Moreover, if $n_{\mathrm{D}}$ is a unit normal vector to $\mathrm{D}\left(r_{0}\right)$, then along any radial geodesic $\gamma=\gamma(r)$,

$$
\left\langle R_{\alpha}(r), n_{\mathrm{D}}(r)\right\rangle \geq 1-B r-\frac{1}{2} A r^{2} .
$$

To carry out Step II we first make an observation about contactizations of Liouville domains and exact symplectic manifolds. For the remainder of this section $\left(W, \beta_{0}\right)$ will be a Liouville domain. Let $\mu$ denote the restriction of $\beta_{0}$ to $\partial W$. By definition $\mu$ is a contact form. The completion of $W$ is obtained as usual by adding the cylindrical end $[1, \infty) \times \partial W$ equipped with the Liouville form $t \mu$, where $t$ is the "radial" coordinate on $[1, \infty)$. The resulting manifold will be denoted $W_{\infty}$, and we will also denote this extended 1 -form by $\beta_{0}$. For any constant $a>1$ we set $W_{a}=W \cup([1, a) \times \partial W)$. We say an almost complex structure is adapted to $\beta_{0}$ if

(a) it is tamed by $d \beta_{0}$,

(b) it preserves the contact structure ker $\alpha$ on each $\{t\} \times \partial W$, and

(c) it sends $\partial_{t}$, point-wise, to some positive multiple of the Reeb field $R_{\mu}$.

Recall that a 2 -form $\omega$ tames an almost complex structure $J$ if $\omega(u, J u)>0$ for any non-zero vector $u$. Note that $\omega$ is then automatically non-degenerate since any $u$ in the kernel of $\omega$ would violate the taming condition.

Proposition 4.3 (proved in Section 4.2). Suppose $\beta_{1}$ is a 1 -form on $W_{T}$ (for some $T>1$ ) such that $d \beta_{1}$ is a symplectic form on $W_{T}$ and there is an almost complex structure which is adapted to $\beta_{0}$ and tamed by $d \beta_{1}$. Then, for any $T_{0} \in[1, T)$, the contactization of $\left(\bar{W}_{T_{0}}, \beta_{1}\right)$ embeds in the contactization of $\left(W_{\infty}, \beta_{0}\right)$.

In our situation, we want to apply the above proposition to the complex structure on $\mathrm{D}(r)$ obtained by pushing forward, via $\exp _{p}$, some complex structure on $\xi_{p}$ tamed by $d \alpha_{p}$. 
Proposition 4.4 (proved in Section 4.3). Given a compatible metric structure $(\alpha, g, J)$ on the contact manifold $(M, \xi)$, with sectional curvature bounded as in (4.1), the complex structure $\left(\exp _{p}^{\xi}\right)_{*} J_{p}$ is tamed by the restriction of da to $\mathrm{D}(r)$ whenever

$$
r<\min \left(r_{\pitchfork}, \frac{1}{(1+2 n(n-1)) \bar{H}}\right),
$$

where the constants are defined at the beginning of this section.

The previous two propositions will guarantee that the pull-back of the contact structure on $\mathrm{C}(r)$ via $E$ will be standard, that is, embed in the standard contact structure on $\mathbb{R}^{2 n+1}$, thus completing Step II. So we are left to complete Step III by estimating the size of a geodesic ball that can be embedded in such a cylinder. We can make such an estimate in a more general context that does not involve anything from the special geometry of compatible metrics except that the Reeb field is geodesic.

Proposition 4.5 (proved in Section 4.4). Let $(M, g)$ be a complete Riemannian manifold whose sectional curvature is bounded above by $K$. Let $X$ be a unit norm geodesic vector field on $M$ and $p$ a point in $M$. Consider the disk

$$
\mathrm{D}\left(r_{0}\right):=\left\{\exp _{p}(v): v \in X_{p}^{\perp},\|v\|<r_{0}\right\} \quad \text { with } \quad r_{0}<\min \left(\frac{\operatorname{inj}(g)}{2}, \frac{\pi}{2 \sqrt{K}}\right) .
$$

We denote by $n$ a unit vector field positively transverse to $\mathrm{D}\left(r_{0}\right)$ and assume we have the following estimate along a radial geodesic $\gamma$ :

$$
\langle X(\gamma(r)), n(\gamma(r))\rangle \geq 1-P(r)
$$

where $P=P(r) \geq 0$ depends only on the distance $r$ to $p$ and $P(r) \leq 1$ on $\left[0, r_{0}\right]$. Then the cylinder $\mathrm{C}\left(r_{0}\right)=\Phi\left((-\infty, \infty) \times \mathrm{D}\left(r_{0}\right)\right)$ given by the flow $\Phi$ of $X$ contains a geodesic ball of radius

$$
\operatorname{sn}_{K}^{-1}\left(\left(1-P\left(r_{0}\right)\right) \operatorname{sn}_{K}\left(r_{0}\right)\right)
$$

about $p$.

We can now prove Theorem 4.1 estimating the size of a Darboux ball.

Proof of Theorem 4.1. By Proposition 4.2, if $r<r_{\pitchfork}$, then $\mathrm{D}(r)$ is embedded in $M$ and the Reeb vector field $R_{\alpha}$ is transverse to $\mathrm{D}(r)$. Since $R_{\alpha}$ is transverse to $\mathrm{D}(r)$ the restriction of the contact form $\alpha$ to $\mathrm{D}(r)$ is a primitive for an exact symplectic form $d \alpha$ on $\mathrm{D}(r)$.

Let $\beta$ denote the pull-back of $\left.\alpha\right|_{\mathrm{D}(r)}$ to $D_{\xi}(r)$ by the exponential map. The contactization of $\left(D_{\xi}(r), \beta\right)$ is the contact structure on $\mathbb{R} \times D_{\xi}(r)$ coming from the contact form $d t+\beta$, and $E: \mathbb{R} \times D_{\xi}(r) \rightarrow M$ is a contact immersion. In fact $E^{*} \alpha=d t+\beta$ since $d E\left(\partial_{t}\right)=R_{\alpha}, \mathcal{L}_{R_{\alpha}} \alpha=0$, and $\alpha\left(R_{\alpha}\right)=1$; thus $\xi=$ ker $\alpha$ lifts to $\operatorname{ker}(d t+\beta)$.

The bound of Proposition 4.4 implies that if

$$
r<r_{\tau}:=\min \left(r_{\pitchfork}, \frac{1}{(1+2 n(n-1)) \bar{H}}\right),
$$


then $d \alpha$ on $\mathrm{D}(r)$ is tamed by $J=\left(\exp _{p}^{\xi}\right)_{*} J_{p}$. Thus $\beta=\left.\left(\exp _{p}^{\xi}\right)^{*} \alpha\right|_{\mathrm{D}(r)}$ on $D_{\xi}(r) \subset \xi_{p}$ is tamed by $J_{p}$. Of course the standard Liouville form $\lambda=\sum_{i=1}^{n} y_{i} d x_{i}$ is adapted to $J_{p}$. Thus by Proposition 4.3 the contactization of any open subdomain of $D_{\xi}(r)$ will contact embed in the contactization of $\left(\mathbb{R}^{2 n}, \lambda\right)$, that is, in the standard contact structure on $\mathbb{R}^{2 n+1}$.

We are left to estimate the maximal size of a geodesic ball $B_{p}(r)$ about $p$ that can be embedded inside the cylinder $\mathrm{C}(r)$ (which of course can then be lifted via $E$ to the contactization of $\left(D_{\xi}(r), \beta\right)$, as above.) Setting $P(r)=B r+\frac{1}{2} A r^{2}$ we see from equation (4.8) that

$$
\left\langle R_{\alpha}(r), n_{\mathrm{D}}(r)\right\rangle \geq 1-P(r),
$$

and Proposition 4.5 allows us to embed a geodesic ball of radius $Q\left(r_{\tau}\right)$ inside the cylinder $\mathrm{C}\left(r_{\tau}\right)$; hence this ball is standard.

4.1. Twisting estimates. Throughout this subsection we will assume that $r \in$ $\left[0, r_{\max }\right)$ and use the notation established at the beginning of Section 4 . Our goal is to prove Proposition 4.2 which estimates, along a radial geodesic, the angle $\left\langle R_{\alpha}, n_{\mathrm{D}}\right\rangle$ between the Reeb vector field and the disks $\mathrm{D}(r)$ as

$$
\left\langle R_{\alpha}, n_{\mathrm{D}}\right\rangle \geq 1-B r-\frac{1}{2} A r^{2}
$$

where $n_{\mathrm{D}}$ is the unit normal vector to $\mathrm{D}(r)$ which coincides with $R_{\alpha}$ at $p$.

First notice that since $r_{\max }$ is less than the injectivity radius of $g$, the disk $\mathrm{D}(r)$ is embedded for any $r \leq r_{\max }$. Next we show that the estimate above implies the transversality result in the proposition. The Reeb field $R_{\alpha}$ is transverse to $\mathrm{D}(r)$ as long as $\left\langle R_{\alpha}, n_{\mathrm{D}}\right\rangle$ is positive. Because the roots of $A t^{2}+2 B t-2$ are $\left(-B \pm \sqrt{2 A+B^{2}}\right) / A$, this is guaranteed whenever $r$ is less than $\frac{-B+\sqrt{2 A+B^{2}}}{A}=$ $\frac{2}{\sqrt{2 A+B^{2}}+B}>0$. Then, we obtain

$$
\min \left(r_{\max }, \frac{2}{\sqrt{2 A+B^{2}}+B}\right)=r_{\text {巾. }} .
$$

We now establish the estimate on $\left\langle R_{\alpha}, n_{\mathrm{D}}\right\rangle$. Let $q$ be any point of $\mathrm{D}(r)$ at some distance $r_{q}$ from $p$. We denote by $\gamma$ the radial geodesic between $p$ and $q$. Estimating along $\gamma$ we have

$$
\begin{aligned}
\left|\left\langle R_{\alpha}, n_{\mathrm{D}}\right\rangle_{q}-1\right| & =\left|\left\langle R_{\alpha}, n_{\mathrm{D}}\right\rangle_{q}-\left\langle R_{\alpha}, n_{\mathrm{D}}\right\rangle_{p}\right| \\
& =\left|\int_{0}^{r_{q}} \frac{d}{d s}\left\langle R_{\alpha}, n_{\mathrm{D}}\right\rangle_{\gamma(s)} d s\right|=\left|\int_{0}^{r_{q}} \nabla_{\dot{\gamma}}\left\langle R_{\alpha}, n_{\mathrm{D}}\right\rangle d s\right| \\
& \leq \int_{0}^{r_{q}}\left|\left\langle\nabla_{\dot{\gamma}} R_{\alpha}, n_{\mathrm{D}}\right\rangle\right|+\left|\left\langle R_{\alpha}, \nabla_{\dot{\gamma}} n_{\mathrm{D}}\right\rangle\right| d s .
\end{aligned}
$$

In the first term, $\left|\left\langle\nabla_{\dot{\gamma}} R_{\alpha}, n_{\mathrm{D}}\right\rangle\right|$ is less than or equal to $\left\|\nabla_{\dot{\gamma}} R_{\alpha}\right\|$, and thus equation (2.21) from Proposition 2.11 gives

$$
\left|\left\langle\nabla_{\dot{\gamma}} R_{\alpha}, n_{\mathrm{D}}\right\rangle\right| \leq B
$$

Hence we are left to estimate $\left\langle R_{\alpha}(s), \nabla_{\dot{\gamma}} n_{\mathrm{D}}(s)\right\rangle$ for all $s \in\left[0, r_{q}\right]$. We now fix such an $s$. Because $s \leq r_{q}$ is less than the injectivity radius, the Gauss lemma guarantees the differential of the exponential map at $p$ gives us an isomorphism between the orthogonal complement of $\dot{\gamma}(0)$ in $T_{p} M$ and the orthogonal complement of $\dot{\gamma}(s)$ in $T_{\gamma(s)} M$. Using this and the fact that $\mathrm{D}(r)$ is the image of $D_{\xi}(r)$ under the 
exponential map one can construct Jacobi fields $J_{1}, \ldots, J_{2 n-1}$ along $\gamma$ such that

(i) $J_{i}(0)=0$ and $J_{i}^{\prime}(0)$ is in $\xi_{p}$,

(ii) all $J_{i}$ are tangent to $D$, and

(iii) $\left\{n_{\mathrm{D}}, \dot{\gamma}, J_{1}, \ldots, J_{2 n-1}\right\}$ is an orthonormal basis at $\gamma(s)$ (but not, a priori, at any other $\gamma(r))$.

We now derive an estimate on the derivative of the Jacobi fields that will be needed below.

Lemma 4.6. With the notation as above, let $J$ be any of the $J_{i}$ Jacobi fields. Then

$$
\left|\left\langle n_{\mathrm{D}}, J^{\prime}(s)\right\rangle\right| \leq \frac{4}{3}|\sec (g)| s .
$$

Proof. Integrating the Jacobi equations $J^{\prime \prime}+R\left(J, \gamma^{\prime}\right) \gamma^{\prime}=0$ component-wise in a parallel moving frame along $\gamma$, one obtains $J^{\prime}(s)=J^{\prime}(0)-\int_{0}^{s} R\left(J(t), \gamma^{\prime}(t)\right) \gamma^{\prime}(t) d t$ where the integral is again component-wise integration in a parallel frame. Hence, using $\left\langle n_{\mathrm{D}}, J^{\prime}(0)\right\rangle=0$ and the bound $|R| \leq \frac{4}{3}|\sec (g)|$ (see, for example, [5, p. 95]) one obtains

$$
\left|\left\langle n_{\mathrm{D}}, J^{\prime}(s)\right\rangle\right| \leq \frac{4}{3}|\sec (g)| \max _{t}\|J(t)\| s .
$$

We claim that for $s \leq \min \left(\operatorname{inj}(g), \frac{\pi}{2 \sqrt{K}}\right)$ the function $\|J(t)\|$ is increasing on the interval $[0, s]$. Indeed, if $r$ is the distance function from $p$ and $\nabla^{2} r$ denotes its Hessian, then a simple computation (or see [20, Lemma III.4.10, p. 109]) coupled with Proposition 3.1 yields

$$
\begin{aligned}
\frac{\partial}{\partial t}\|J(t)\|^{2} & =2 g\left(\nabla_{\dot{\gamma}(t)} J(t), J(t)\right)=2 \nabla^{2} \mathrm{r}(J(t), J(t)) \\
& \geq\left(\operatorname{ct}_{K} \circ \mathrm{r}(\gamma(t))\|J(t)\|^{2}=\operatorname{ct}_{K}(t)\|J(t)\|^{2},\right.
\end{aligned}
$$

and the length of $J(t)$ must increase until the first zero of $\operatorname{ct}_{K}(t)$, which occurs at $t=\frac{\pi}{2 \sqrt{K}}$ for $K>0$ and does not exist otherwise. Because $\|J(t)\|^{2}$ is increasing, $\|J(t)\| \leq\|J(s)\|=1$ and inequality (4.14) simplifies to the promised estimate (4.13).

At the point $\gamma(s)$, we can decompose the Reeb field as $R_{\alpha}=R_{\gamma} \dot{\gamma}+R_{n} n_{\mathrm{D}}+$ $\sum R_{i} J_{i}$. The first two terms do not contribute to the scalar product with $\nabla_{\dot{\gamma}} n_{\mathrm{D}}$ since $n_{\mathrm{D}}$ is normal to $\gamma$ and $\gamma$ is a geodesic. We can now estimate

$$
\left|\left\langle R_{\alpha}, \nabla_{\dot{\gamma}} n_{\mathrm{D}}\right\rangle(\gamma(s))\right| \leq \sum_{i=1}^{2 n-1}\left|R_{i}\left\langle J_{i}(s), \nabla_{\dot{\gamma}} n_{\mathrm{D}}\right\rangle\right|=\sum_{i=1}^{2 n-1}\left|R_{i}\left\langle\nabla_{\dot{\gamma}} J_{i}(s), n_{\mathrm{D}}\right\rangle\right|,
$$

where the last equality follows because $\left\langle J_{i}(t), n_{\mathrm{D}}\right\rangle=0$ for all $t$.

Notice that $R_{\gamma}^{2}+R_{n}^{2}+\sum R_{i}^{2}=\left\|R_{\alpha}\right\|^{2}=1$ so $\sum R_{i}^{2} \leq 1$. Equation (4.13) from Lemma 4.6 and the Cauchy-Schwarz inequality give

$$
\left|\left\langle R_{\alpha}, \nabla_{\dot{\gamma}} n_{\mathrm{D}}\right\rangle(q)\right| \leq \sqrt{2 n-1} \frac{4}{3}|\sec (g)| s .
$$


Using this, equation (4.11), and equation (4.12) we see that

$$
\begin{aligned}
\left|\left\langle R_{\alpha}, n_{\mathrm{D}}\right\rangle_{q}-1\right| & \leq \int_{0}^{r_{q}}\left|\left\langle\nabla_{\dot{\gamma}} R_{\alpha}, n_{\mathrm{D}}\right\rangle\right|+\left|\left\langle R_{\alpha}, \nabla_{\dot{\gamma}} n_{\mathrm{D}}\right\rangle\right| d s \\
& \leq B r_{q}+\left(\sqrt{2 n-1} \frac{4}{3}|\sec (g)|\right) \frac{r_{q}^{2}}{2} \\
& =B r_{q}+\frac{1}{2} A r_{q}^{2},
\end{aligned}
$$

from which equation (4.8) easily follows.

4.2. Embedding contactizations. This subsection contains a proof of Proposition 4.3. We begin with a simple lemma about embedding contactizations. Throughout this subsection we will be using notation established at the beginning of the section.

Lemma 4.7 (Interpolation lemma). Let $\left(W, \beta_{0}\right)$ be a Liouville domain and $\left(W_{\infty}, \beta_{0}\right)$ its completion. Suppose $\beta_{1}$ is a 1 -form on $W_{T}$ (for some $T>1$ ) such that $d \beta_{1}$ is a symplectic form on $W_{T}$ and there is an almost complex structure which is adapted to $\beta_{0}$ and tamed by $d \beta_{1}$. Then for any $T_{0} \in[1, T)$ there is a positive constant $\lambda$ and a Liouville form $\widehat{\beta}$ on $W_{\infty}$ such that

(i) $\widehat{\beta}=\lambda \beta_{0}$ outside $W_{T}$,

(ii) $\widehat{\beta}=\beta_{1}$ on $W_{T_{0}}$, and

(iii) $d \widehat{\beta}$ is tamed by $J$.

Proof. We set $\widehat{\beta}=\rho \beta_{1}+(1-\rho) \lambda \beta_{0}$ where $\rho$ is a function with support in $W_{T}$, equals one on a neighborhood of $W_{T_{0}}$ and which, inside $[1, \infty) \times \partial W$, depends only on $t$ and is non-increasing.

The first two properties of $\widehat{\beta}$ are obvious from its definition, so we are left to show that $\lambda$ and $\rho$ can be chosen so that the third property holds. Notice that for this we can restrict our attention to $[1, \infty) \times \partial W$ where we have

$$
d \widehat{\beta}=\left(-\rho^{\prime}\right) \lambda t d t \wedge \mu+\rho^{\prime} d t \wedge \beta_{1}+\rho d \beta_{1}+(1-\rho) \lambda d \beta_{0} .
$$

(Recall that $\mu$ is the contact form induced on $\partial W$ by $\beta_{0}$.) By the hypothesis $-\rho^{\prime}$ is non-negative. Moreover $d t \wedge \mu(u, J u)$ is non-negative because $J$ is adapted to $\beta_{0}$. So we are left to prove that, using carefully chosen $\rho$ and $\lambda$, for any non-zero $u$ the function

$$
G(u):=\left(\rho^{\prime} d t \wedge \beta_{1}+\rho d \beta_{1}+(1-\rho) \lambda d \beta_{0}\right)(u, J u)
$$

is positive.

Because $d \beta_{0}$ and $d \beta_{1}$ tame $J$, there are positive constants $C_{0}, C_{1}$ and $C$ such that

$$
\frac{1}{\|u\|^{2}} G(u) \geq-\left|\rho^{\prime}\right| C+\rho C_{1}+\lambda(1-\rho) C_{0} .
$$

We cut the interval $\left[T_{0}, T\right)$ into two halves. On the first interval, $\rho$ will be almost 1 and $\rho^{\prime}$ will be almost 0 , and on the second interval $\rho$ will decrease to zero. More precisely, we choose $\rho$ such that

$$
\rho(t) \geq 1-\varepsilon \text { and }\left|\rho^{\prime}(t)\right| \leq \frac{2 \varepsilon}{\left(T-T_{0}\right) / 2}, \quad \text { for } t \in\left[T_{0},\left(T_{0}+T\right) / 2\right],
$$


and

$$
\rho(t) \leq 1-\varepsilon \text { and }\left|\rho^{\prime}(t)\right| \leq \frac{2(1-\varepsilon)}{\left(T-T_{0}\right) / 2}, \quad \text { for } t \in\left[\left(T_{0}+T\right) / 2, T\right] .
$$

On the first subinterval, one has

$$
-C\left|\rho^{\prime}\right|+\rho C_{1} \geq-C \frac{2 \varepsilon}{\left(T-T_{0}\right) / 2}+(1-\varepsilon) C_{1}
$$

which is positive if $\varepsilon$ is sufficiently small. On the second interval

$$
-C\left|\rho^{\prime}\right|+\lambda(1-\rho) C_{0} \geq-C \frac{2(1-\varepsilon)}{\left(T-T_{0}\right) / 2}+\lambda \varepsilon C_{0},
$$

which, for a fixed positive $\epsilon$, is positive if $\lambda$ is sufficiently large.

We now consider $\widehat{\beta}$ as in the above lemma. The contactization of $\left(W_{T_{0}}, \beta_{1}\right)$ embeds into the contactization of $\left(W_{\infty}, \widehat{\beta}\right)$. Since the contactizations of $\beta_{0}$ and $\lambda \beta_{0}$ are isomorphic, the following lemma finishes the proof of Proposition 4.3 .

Lemma 4.8. The contactizations of $\widehat{\beta}$ and $\lambda \beta_{0}$ are isomorphic.

Proof. Consider the path of 1 -forms $\lambda_{s}=(1-s) \lambda \beta_{0}+s \widehat{\beta}$. The preceding lemma says that $d \lambda_{s}$ tame $J$ for all $s$ and thus are all symplectic forms on $W_{\infty}$. Thus $\alpha_{s}=d t+\lambda_{s}$ is a path of contact forms. Moser's technique provides an isotopy that connects the corresponding contact structures if the vector fields constructed in Moser's technique can be integrated for a sufficiently long time. It is clear that it can be so integrated since $\beta_{0}$ and $\widehat{\beta}$ coincide outside the compact set $\overline{W_{T}}$.

4.3. Taming $J$. In this subsection we prove Proposition 4.4. We begin with a general lemma that holds in any Riemannian manifold. We will need the auxiliary functions

$$
H_{1}(r)=\sqrt{1+\left(\frac{\operatorname{sn}_{\kappa}(r)}{r}\right)^{2}} \text { and } H_{2}(r)=\frac{4}{3}|\sec (g)|\left(r H_{1}(r)+\int_{0}^{r} H_{1}(t) d t\right) .
$$

Lemma 4.9. Suppose the sectional curvature of $(M, g)$ is bounded below by some constant $\kappa$. Let $\gamma:[0, R] \rightarrow M$ be a unit speed geodesic with $R<\operatorname{inj}(g)$ and set $p=\gamma(0)$. Let $X(r)=\left(d \exp _{p}\right)_{r \gamma^{\prime}(0)} v$ be a vector field along $\gamma$ obtained as the image of a fixed unit vector $v \in T_{p} M$. (We think of $v$ as a vector in $T_{w}\left(T_{p}(M)\right.$ ) for every $w \in T_{p} M$ using the canonical identification of a vector space with its tangent space at any point.) Then, using notation from equation (4.15) we have

$$
\|X(r)\| \leq H_{1}(r)
$$

and

$$
\left\|\nabla_{\dot{\gamma}} X(r)\right\| \leq H_{2}(r),
$$

for $r \in\left[0, r_{\max }\right)$ where $r_{\max }$ was defined in equation (4.2).

Proof. Let $J$ be the Jacobi field along $\gamma$ which satisfies $J(0)=0$ and $J^{\prime}(0)=X(0)$. According to [5. Theorem II.7.1, p. 88], one has $J(r)=r X(r)$. One can decompose $X$ into $X^{\top}$ which is parallel to $\dot{\gamma}$ and $X^{\perp}$ which is perpendicular to $\dot{\gamma}$. Then by the Gauss lemma, one has $\left\|X^{\top}(r)\right\|=\left\|X^{\top}(0)\right\|$. The perpendicular part is 
estimated by Rauch's theorem [5, Theorem IX.2.3, p. 390], which gives $\left\|X^{\perp}(r)\right\| \leq$ $\operatorname{sn}_{\kappa}(r) / r\left\|X^{\perp}(0)\right\|$. Thus we have

$$
\begin{aligned}
\|X(r)\|^{2} & =\left\|X^{\top}(r)\right\|^{2}+\left\|X^{\perp}(r)\right\|^{2} \\
& \leq 1+\frac{\operatorname{sn}_{\kappa}(r)^{2}}{r^{2}} .
\end{aligned}
$$

We will now establish equation (4.17). Since $X=r^{-1} J$, we have $X^{\prime}=r^{-1}\left(J^{\prime}-X\right)$. The Jacobi equation for $J$ reads $J^{\prime \prime}+R J=0$ where $R J$ is shorthand for $R\left(J, \gamma^{\prime}\right) \gamma^{\prime}$. In a parallel frame along $\gamma$, the components of this equation can be integrated component-wise. We can now estimate

$$
\begin{aligned}
\left\|X^{\prime}(r)\right\| & =r^{-1}\left\|J^{\prime}(r)-X(r)\right\|=r^{-1}\left\|\int_{0}^{r}-R J-X^{\prime}\right\| \leq r^{-1} \int_{0}^{r}\|R J\|+\left\|X^{\prime}\right\| \\
& \leq \frac{4}{3}|\sec (g)| \max \|J\|+r^{-1} \int_{0}^{r}\left\|X^{\prime}\right\| \\
& \leq \frac{4}{3}|\sec (g)| r H_{1}(r)+\frac{1}{r} \int_{0}^{r}\left\|X^{\prime}\right\|,
\end{aligned}
$$

where the second inequality follows from [5, p. 95] just as in the proof of Lemma 4.6. So estimating $\left\|X^{\prime}\right\|$ is now a Grönwall type problem. We set $f(r)=\left\|X^{\prime}(r)\right\|$ and $\alpha(r)=\frac{4}{3}|\sec (g)| r H_{1}(r)$, so that the above inequality reads

$$
f(r) \leq \alpha(r)+\frac{1}{r} \int_{0}^{r} f(t) d t
$$

Setting $v(r)=\frac{1}{r} \int_{0}^{r} f(t) d t$ and keeping in mind that $f$ is smooth and $f(0)=0$, we have

$$
v^{\prime}(r)=\frac{1}{r}\left(f-\frac{1}{r} \int_{0}^{r} f(t) d t\right) \leq \alpha(r) / r .
$$

Since $v(0)=0$, we see that $v(r) \leq \int_{0}^{r} \frac{\alpha(u)}{u} d u$, which can be substituted into equation (4.18) to obtain the announced estimate.

Lemma 4.10. Given a radial geodesic $\gamma$ in $\mathrm{D}\left(r_{0}\right)$ starting at $p=\gamma(0)$, consider two vector fields $X$ and $Y$ along $\gamma=\gamma(r)$ which are the images of unit vectors in $\xi_{p}$ under the differential of $\exp _{p}$ as described in Lemma 4.9. Then the derivative of the function $F(r)=\left(1 / \theta^{\prime}\right) d \alpha(X, Y)$ is bounded by the constant $\bar{H}$ defined in equation (4.4), i.e.

$$
\left|F^{\prime}(r)\right| \leq \bar{H}, \quad \text { for } \quad r \in\left[0, r_{\max }\right]
$$

where $r_{\max }$ is defined in equation (4.2).

Proof. By Proposition 2.3 the metric $g$ can be expressed as

$$
g(X, Y)=\frac{1}{\theta^{\prime}} d \alpha(X, \phi(Y))+\alpha(X) \alpha(Y)
$$

therefore

$$
\frac{1}{\theta^{\prime}} d \alpha(X, Y)=-\langle X, \phi(Y)\rangle
$$


and

$$
\frac{d}{d r} F(r)=-\left\langle\nabla_{r} X, \phi(Y)\right\rangle-\left\langle X, \phi\left(\nabla_{r} Y\right)\right\rangle-\left\langle X,\left(\nabla_{r} \phi\right)(Y)\right\rangle .
$$

So Lemma 4.9 and estimate (2.22) yield

$$
\begin{aligned}
\left|\frac{d}{d r} F(r)\right| & \leq H_{2}\|\phi(Y)\|+H_{2}\|X\|+(\|[J, J]\| / 2+2 B)\|X\| \cdot\|Y\| \\
& \leq 2 H_{1} H_{2}+(\|[J, J]\| / 2+2 B) H_{1}^{2} .
\end{aligned}
$$

Note that $H_{1}(r)$ is increasing when $\kappa<0$, constant (equal to $\sqrt{2}$ ) for $\kappa=0$, and decreasing on $\left[0, \frac{\pi}{2 \sqrt{\kappa}}\right]$ to 1 at $\frac{\pi}{2 \sqrt{\kappa}}$ if $\kappa>0$. Also the function $H_{2}(r)$ is increasing and vanishing at $r=0$. Thus, on the interval [ $\left.0, r_{\max }\right)$ where $H_{1}$ and $H_{2}$ are defined we have the following simple estimates:

$$
\begin{aligned}
& H_{1}(r) \leq \bar{H}_{1}:=\max _{\left[0, r_{\max }\right)} H_{1}(r)= \begin{cases}H_{1}(0), & \text { if } \kappa \geq 0, \\
H_{1}\left(r_{\max }\right), & \text { if } \kappa<0,\end{cases} \\
& H_{2}(r) \leq \bar{H}_{2}=\frac{8}{3}|\sec (g)| \bar{H}_{1} r_{\max } .
\end{aligned}
$$

In addition both $H_{1}$ and $H_{2}$ are non-negative functions, hence $\left|F^{\prime}(r)\right| \leq \bar{H}$.

Proof of Proposition 4.4. Given any non-zero vector $u$ tangent to $D(r)$ at some point $q$ let $\gamma$ be the geodesic from $p$ to $q$. Choose a symplectic basis $X_{1}, \ldots, X_{n}$, $Y_{1}, \ldots, Y_{n}$ of $\xi$ at $p$ with $Y_{i}=J X_{i}$ and $\left|X_{i}\right|=1$ and use the same notation for the vector fields along $\gamma$ obtained using $\exp _{p}$ as in Lemma 4.9. This gives a moving frame for $D(r)$. Hence there are constants $a_{i}$ and $b_{i}$ such that $u=\sum_{i} a_{i} X_{i}+b_{i} Y_{i}$. Then

$$
\begin{aligned}
& d \alpha(u, J u)= \sum_{1 \leq i, j \leq n}\left(-a_{i} b_{j} d \alpha\left(X_{i}, X_{j}\right)+a_{i} a_{j} d \alpha\left(X_{i}, Y_{j}\right)\right. \\
&\left.-b_{i} b_{j} d \alpha\left(Y_{i}, X_{j}\right)+a_{j} b_{i} d \alpha\left(Y_{i}, Y_{j}\right)\right) \\
&=\sum_{i=1}^{n}\left(a_{i}^{2}+b_{i}^{2}\right) d \alpha\left(X_{i}, Y_{i}\right)+\sum_{1 \leq i \neq j \leq n}\left(-a_{i} b_{j} d \alpha\left(X_{i}, X_{j}\right)+a_{i} a_{j} d \alpha\left(X_{i}, Y_{j}\right)\right. \\
&\left.-b_{i} b_{j} d \alpha\left(Y_{i}, X_{j}\right)+a_{j} b_{i} d \alpha\left(Y_{i}, Y_{j}\right)\right) .
\end{aligned}
$$

At $r=0,\left(1 / \theta^{\prime}\right) d \alpha\left(X_{i}, Y_{i}\right)=1$, and $d \alpha(Z, W)=0$ if $Z$ and $W$ are any pair of vectors appearing in the second sum above. From the derivative estimate in equation (4.19) we see that

$$
\frac{1}{\theta^{\prime}} d \alpha\left(X_{i}, Y_{i}\right) \geq 1-\bar{H} r \quad \text { and } \quad \frac{1}{\theta^{\prime}}|d \alpha(Z, W)|(r) \leq \bar{H} r .
$$

To continue the computation, extend the $X_{i}, Y_{i}$ and $n_{\mathrm{D}}$ to a neighborhood of $\gamma$ and use them to define an auxiliary metric on the neighborhood so that they form an orthonormal basis. In this metric the norm squared of $u$, which we denote by $N(u)$, is $\sum_{i=1}^{n}\left(a_{i}^{2}+b_{i}^{2}\right)$. We now have

$$
\frac{1}{\theta^{\prime}} d \alpha(u, J u) \geq(1-\bar{H} r) N(u)-r \bar{H} \sum_{1 \leq i \neq j \leq n}\left(\left|a_{i}\right|\left|b_{j}\right|+\left|a_{i}\right|\left|a_{j}\right|+\left|b_{i}\right|\left|b_{j}\right|+\left|a_{j}\right|\left|b_{i}\right|\right) .
$$


Since there are $2\left(\begin{array}{c}n \\ 2\end{array}\right)=n(n-1)$ terms in the sum and $\frac{1}{2} N(u) \geq\left|a_{*}\right|\left|b_{*}\right|\left(\right.$ or $\left|a_{*}\right|\left|a_{*}\right|$ or $\left.\left|b_{*}\right|\left|b_{*}\right|\right)$, we see that

$$
\frac{1}{\theta^{\prime}} d \alpha(u, J u) \geq(1-(1+2 n(n-1)) \bar{H} r) N(u) .
$$

Hence, for $u \neq 0$ we have $d \alpha(u, J u)>0$ if $r<((1+2 n(n-1)) \bar{H})^{-1}$.

4.4. From cylinders to balls. The goal of this subsection is to prove Proposition 4.5 which guarantees that Reeb flow cylinders contain embedded geodesic balls of a certain radius.

Proof of Proposition 4.5. Recalling the hypothesis of the proposition we have a complete Riemannian manifold $(M, g)$ whose sectional curvatures are bounded above by $K$ and a number $r_{0}$ such that

$$
r_{0}<\min \left(\frac{\operatorname{inj}(g)}{2}, \frac{\pi}{2 \sqrt{K}}\right) .
$$

The vector field $X$ is a unit speed geodesic vector field on $M$ and $p$ is some point in $M$. Let $\xi$ be the hyperplane field $X_{p}^{\perp}$. We set $D_{\xi}\left(r_{0}\right)=\left\{v \in X_{p}^{\perp},\|v\|<r_{0}\right\}$ and

$$
\mathrm{D}\left(r_{0}\right)=\left\{\exp _{p}(v): v \in D_{\xi}\left(r_{0}\right)\right\} .
$$

Moreover, we set

$$
E: \mathbb{R} \times D_{X_{p}}\left(r_{0}\right) \rightarrow M:(t, v) \mapsto \Phi\left(t, \exp _{p}(v)\right),
$$

where $\Phi$ is the flow of $X$. We finally set $\mathrm{C}\left(r_{0}\right)=$ image $(E)$.

Denote by $\mathrm{B}^{\text {conv }}$ the ball at $p$ of radius $r_{0}$, and $\mathrm{C}^{\text {conv }}\left(r_{0}\right)=\mathrm{C}\left(r_{0}\right) \cap \mathrm{B}^{\text {conv }}$ the portion of $\mathrm{C}\left(r_{0}\right)$ in $\mathrm{B}^{\text {conv }}$. Note that Proposition 3.1 implies that the sphere $\partial \mathrm{B}^{\text {conv }}$ is convex. Let $B$ be the connected component of $E^{-1}\left(\mathrm{C}^{\operatorname{conv}}\left(r_{0}\right)\right)$ that contains $(0,0)$. Since $r_{0}<\operatorname{conv}(g)$ we observe that $E$ restricted to $B$ is an embedding. To see this we need to study the structure of $B$. We first notice that $B \cap \xi_{p}$ is $D_{\xi}\left(r_{0}\right)$, the ball of radius $r_{0}$ in $\xi_{p}$. Now for each $x \in D_{\xi}\left(r_{0}\right)$ let $I_{x}=(\mathbb{R} \times\{x\}) \cap B$. So $B=\bigcup_{x \in D_{\xi}\left(r_{0}\right)} I_{x}$. Each $I_{x}$ clearly contains the origin $(i . e .(0, x))$. We moreover claim that it is a connected interval for all $x$. If not, then there is some $x_{0}$ for which it is not connected. Let $\gamma$ be the geodesic in $\mathrm{D}\left(r_{0}\right)$ from $p$ to $x_{0}$. Let $J_{x}$ be the smallest interval containing $I_{x}$ for each $x \in \gamma$ and $K_{x}$ the image of $x$ under the flow of $X$ for times in $J_{x}$. Finally the union of all $K_{x}$ for $x \in \gamma$ is a disk $A$. By hypothesis $A$ is not contained in $\mathrm{B}^{\text {conv }}$. If $x^{\prime}$ is the first point on $\gamma$ such that $K_{x^{\prime}}$ is not contained in the interior of $\mathrm{B}^{\text {conv }}$, then $K_{x^{\prime}}$ is a geodesic which has an interior tangency with $\partial \mathrm{B}^{\text {conv }}$. This contradicts the convexity of $\mathrm{B}^{\text {conv }}$. Thus the $I_{x}$ are all connected. This implies that the restriction of $E$ to $B$ is injective because, since we are below the injectivity radius, the ball $\mathrm{B}^{\text {conv }}$ is divided into two connected components by $\mathrm{D}\left(r_{0}\right)$ and, in particular, there is no trajectory of $X$ leaving $\mathrm{D}\left(r_{0}\right)$ and returning to it without leaving $\mathrm{B}^{\text {conv }}$.

Next, we estimate a radius $\bar{r}$ of a convex ball centered at $p$ and contained in $\mathrm{C}^{\text {conv }}\left(r_{0}\right)$; a maximal such ball $B_{p}(\bar{r})$ will either be of radius $\geq r_{0}$ or have a tangency with $\left(\partial \mathrm{C}\left(r_{0}\right)\right) \cap \mathrm{B}^{\text {conv }}$. Let $q$ be a point of the tangency and $v$ be the point of intersection of the orbit of $X$ through $q$ and the disk $\mathrm{D}\left(r_{0}\right)$. Clearly $v \in\left(\partial \mathrm{C}\left(r_{0}\right)\right) \cap \mathrm{B}^{\text {conv }}$ and thus $d(p, v)=r_{0}$. Moreover we know that $d(p, q)=\bar{r}$. Consider the geodesic triangle $T(p, q, v)$ consisting of the unique geodesics connecting these points. So both sides $(p, q)$ and $(p, v)$ of $T(p, q, v)$ are radial geodesics emanating from $p$, and $(q, v)$ is a piece of the orbit of $X$. This piece of orbit is contained in $\mathrm{B}^{\text {conv }}$; otherwise 
it would contradict the convexity of $\partial \mathrm{B}^{\text {conv }}$. So $d(q, v) \leq \operatorname{diam}\left(\mathrm{B}^{\text {conv }}\right)=2 r_{0}$ and the perimeter of $T(p, q, v)$ is less than $4 r_{0}$.

We choose $\gamma(s)$ to be the radial geodesic parameterizing $(p, v)$, i.e. $\gamma(0)=p$ and $\gamma\left(r_{0}\right)=v$. Denote by $0 \leq \phi \leq \frac{\pi}{2}$ the angle between $X\left(\gamma\left(r_{0}\right)\right)$ and the normal $n\left(\gamma\left(r_{0}\right)\right)$, and by $0 \leq \varphi \leq \frac{\pi}{2}$ the angle between $X\left(\gamma\left(r_{0}\right)\right)$ and the line spanned by $\gamma^{\prime}\left(r_{0}\right)$. Observe that $\frac{\pi}{2}-\phi \leq \varphi$; thus using estimate (4.9) we have

$$
\sin (\varphi) \geq \cos (\phi) \geq 1-P\left(r_{0}\right)
$$

Toponogov's theorem (in a slightly unusual curvature upper bound setup; see e.g. [13. Theorem 4.1, p. 197]) allows us to compare with a geodesic triangle $T=$ $T\left(p^{\prime}, q^{\prime}, v^{\prime}\right)$ in the space form $M_{K}$, based at vertex $v^{\prime}$ with angle $\varphi^{\prime}=\varphi$ at $v^{\prime}$, $d\left(p^{\prime}, v^{\prime}\right)=d(p, v)=r_{0}$, and $d\left(q^{\prime}, v^{\prime}\right)=d(q, v)$. The sectional curvature is bounded above by $K$ and the perimeter of $T(p, q, v)$ is less than $\min (2 \operatorname{inj}(g), 2 \pi / \sqrt{K})$, so Toponogov's theorem gives

$$
d\left(p^{\prime}, q^{\prime}\right) \leq d(p, q)=\bar{r} .
$$

Now, let $\theta^{\prime}$ be the angle at the vertex $q^{\prime}$ in the reference triangle. The law of sines [5. Note II.5, p. 103 and references therein] applied to the triangle $T\left(p^{\prime}, q^{\prime}, v^{\prime}\right)$ yields

$$
\frac{\sin \theta^{\prime}}{\operatorname{sn}_{K}\left(r_{0}\right)}=\frac{\sin \varphi}{\operatorname{sn}_{K}\left(d\left(p^{\prime}, q^{\prime}\right)\right)} .
$$

This combines with $\sin \left(\theta^{\prime}\right) \leq 1$ and inequality (4.21) to give

$$
\operatorname{sn}_{K}(\bar{r}) \geq \operatorname{sn}_{K}\left(r_{0}\right)\left(1-P\left(r_{0}\right)\right),
$$

hence the announced bound since the functions $\mathrm{sn}_{K}$ are increasing.

\section{Size OF STANDARD NEIGHBORHOODS IN DIMENSION 3}

In this section we show how to use a geometric method similar to the one in Section 4 to provide a stronger estimate on the Darboux radius in dimension 3. We also show how to use this idea to construct standard neighborhoods of closed geodesics with an estimated size.

In Subsection 5.1 we state two propositions that essentially say if $N=\zeta \times D^{2}$ is a neighborhood of a Reeb orbit and one can control the twisting of the contact structure or its Reeb vector field, then the contact structure on $N$ can be embedded in the standard contact $\mathbb{R}^{3}$. We then prove our main results in dimension 3 using previously derived geometric estimates of Section 4. In the following sections we then prove facts about characteristic foliations that were needed in the proofs of our main result.

5.1. From geometric control to topology. The geometric setup used throughout this section is the following. We consider $(\alpha, g, J)$ a contact metric structure compatible with $(M, \xi)$, and denote by $R_{\alpha}$ its Reeb vector field. Let $\zeta$ be a portion of a geodesic which is either an arc or a circle. We denote by $\nu \zeta$ the normal bundle to $\zeta$ with respect to $g$. We denote by $\exp _{\nu}: \nu \zeta \rightarrow M$ the restriction to $\nu \zeta$ of $\exp : T M \rightarrow M$ (we assume as usual that $g$ is complete).

We fix a positive radius $r$ less than the injectivity radius $\operatorname{inj}(g)$ and consider, for each $z$ in $\zeta$, the embedded disk

$$
\mathrm{D}_{z}:=\exp _{\nu}\left(\left\{v \in \nu_{z} \zeta ;|v| \leq r\right\}\right),
$$


and we denote by $n_{\mathrm{D}}$ the unit vector field orthogonal to all $\mathrm{D}_{z}$ which coincides with $R_{\alpha}$ along $\zeta$.

For each radius $r$ we denote by

$$
\mathrm{T}(r)=\exp _{\nu}(\{v \in \nu \zeta ;|v| \leq r\})
$$

the tube of radius $r$ around $\zeta$. It is the image of either a solid torus or a thickened disk depending on whether $\zeta$ is a circle or an arc, but $\exp _{\nu}$ may fail to be an embedding. The following lemma, which has nothing to do with contact geometry and will be proved at the end of this section, gives a sufficient condition to have an embedding in the thickened disk case.

Lemma 5.1. Suppose the sectional curvature of $g$ is bounded above by $K$ and let $r$ be a positive number such that

$$
r<\min \left(\frac{\operatorname{inj}(g)}{2}, \frac{\pi}{2 \sqrt{K}}\right) .
$$

For every geodesic arc $\zeta$ with length less than $2 r$, the tube $\mathrm{T}(r)$ is embedded.

Returning to the specific setting of contact geometry, we will first need the following result.

Proposition 5.2. If $\zeta$ is a closed Reeb orbit such that $\mathrm{T}(r)$ is embedded and $\xi$ is transverse to $n_{\mathrm{D}}$, then $(\mathrm{T}(r), \xi)$ is contactomorphic to a domain in $\left(S^{1} \times \mathbb{R}^{2}\right.$, $\left.\operatorname{ker}\left(d \phi+\rho^{2} d \theta\right)\right)$, where $\phi$ is the angular coordinate on $S^{1}$ and $(\rho, \theta)$ are polar coordinates on $\mathbb{R}^{2}$. In particular, $(\mathrm{T}(r), \xi)$ is universally tight.

In the case where $\zeta$ is an interval in a Reeb orbit, we will use the embedding criterion above and impose the stronger condition that the Reeb vector field $R_{\alpha}$ stays transverse to all $\mathrm{D}_{z}$. It will allow us to embed $\mathrm{T}(r)$ in a solid torus, which we will then embed in the standard $\left(\mathbb{R}^{3}, \xi_{\text {std }}\right)$.

Proposition 5.3. In the above setup and under the hypothesis of Lemma 5.1, if $\zeta$ is an arc and the Reeb vector field $R_{\alpha}$ is transverse to the disks $\mathrm{D}_{z}$, then $(\mathrm{T}(r), \xi)$ is contactomorphic to a domain in $\left(\mathbb{R}^{3}, \xi_{\mathrm{std}}\right)$.

Now, Theorems 1.7 and 1.8 follow at once.

Proof of Theorem 1.7. By the hypothesis of the theorem and Propositions 4.2 and 5.3 the cylinder $\mathrm{T}(r)$ is embedded and the contact structure on $\mathrm{T}(r)$ is contactomorphic to a domain in $\left(\mathbb{R}^{3}, \xi_{\text {std }}\right)$. Thus any geodesic ball embedded in $\mathrm{T}(r)$ is standard. We will now prove that the geodesic ball $B(p, r)$ of radius $r$ about $p$ is contained in $\mathrm{T}(r)$, and thus the theorem will follow. We denote the boundary of $B(p, r)$ by $S(p, r)$.

Since $\mathrm{T}(r)$ contains very small balls around $p$, there is a positive first radius $r_{0}$ such that the sphere $S\left(p, r_{0}\right)$ intersects $\partial \mathrm{T}(r)$. We denote by $p_{ \pm}=\zeta( \pm r)$ the two extremities of $\zeta$. We denote by $D_{ \pm}$the geodesic disks normal to $\zeta$ centered at $p_{ \pm}$. The boundary of $\mathrm{T}(r)$ is made of $D_{ \pm}$, which we will call horizontal, together with the vertical part made of points at distance $r$ from $\zeta$. One can see that the intersection between $S\left(p, r_{0}\right)$ and $\partial \mathrm{T}(r)$ cannot meet the intersection between the horizontal and vertical parts. At a point in the interior of the vertical part, the plane tangent to $\partial \mathrm{T}(r)$ is the orthogonal to a geodesic which is normal to $\zeta$ (by the generalized Gauss Lemma; see e.g. [10, Lemma 2.11, p. 26]). Likewise, the tangent space of $S\left(p, r_{0}\right)$ at any point is the orthogonal to a geodesic normal to $S\left(p, r_{0}\right)$ and 
going through $p$. So, at a point where $S\left(p, r_{0}\right)$ is tangent to the vertical part of $\partial \mathrm{T}(r)$, those geodesics coincide, and we see that the relevant point of $\zeta$ is $p$. Hence $r_{0}=r$ in this case.

Suppose now that the intersection between $S\left(p, r_{0}\right)$ and $\partial \mathrm{T}(r)$ is in $D_{+}$or $D_{-}$. Let $q$ be a tangency point in one of those disks, say $D_{+}$. Let $\gamma$ be the geodesic from $p_{+}$to $q$ (recall we are below the convexity radius). Note that $\gamma$ is normal to $\zeta$. Let $\gamma^{\prime}$ be the geodesic between $p$ and $q$. Since $S\left(p, r_{0}\right)$ is tangent to $D_{+}$at $q$ the Gauss lemma implies that $\gamma^{\prime}$ is normal to $\gamma \subset D_{+}$. Now there are two cases to consider. If $q=p_{+}$, then $r_{0}=r$ and we have established our claim. If $q \neq p_{+}$, then we contradict Lemma 5.1 applied to $\gamma$, because the tube around $\gamma$ with radius $r$ is not embedded. Indeed we have two geodesics $\zeta$ and $\gamma^{\prime}$ normal to $\gamma$ with length at most $r$ which intersect.

Proof of Theorem 1.8. By the hypothesis of the theorem and Proposition 4.2, the geodesic tube $\mathrm{T}(r)$ about the geodesic $\gamma$ is embedded and the Reeb vector field stays transverse to all the disks $D_{z}$. Since the contact planes are perpendicular to the Reeb vector field, this implies that they are transverse to the vector field $n_{\mathrm{D}}$ normal to the disks. The theorem now follows from Proposition 5.2 .

The next sections are devoted to the proof of Propositions [5.2 and 5.3 but first we come back to Lemma 5.1 .

Proof of Lemma 5.1. By the assumption $r$ is less than the injectivity radius. Thus, the restriction of $\exp _{\nu}$ to $\{z\} \times D(r)$ for every fixed $z$ is injective. Thus injectivity of $\exp _{\nu}$ can fail only when there is a geodesic triangle $T(p, q, v)$ in $\mathrm{T}(r)$ formed by a piece of the geodesic $\zeta$ between $p=\zeta\left(z_{0}\right)$ and $q=\zeta\left(z_{1}\right)$ in $\mathrm{T}(r)$ and two "radial" geodesics $\gamma_{0}$ in $\mathrm{D}_{z_{0}}$, and $\gamma_{1}$ in $\mathrm{D}_{z_{1}}$, such that $\gamma_{0}(0)=p, \gamma_{1}(0)=q$ and $\gamma_{0}\left(t_{0}\right)=\gamma_{1}\left(t_{1}\right)=v$ for some $t_{0}, t_{1} \leq r$. Since $\zeta$ is orthogonal to $\mathrm{D}_{z_{0}}$ and $\mathrm{D}_{z_{1}}$, the triangle $T$ also has two right angles at vertices $p$ and $q$ and its perimeter is at most $4 r$ which is less than $\min (2 \operatorname{inj}(g), 2 \pi / \sqrt{K})$. Toponogov's theorem [13. Theorem 4.1, p. 197] first guarantees there exists an analogous triangle in the model space $M_{K}$. So we already get a contradiction if $K$ is non-positive since there is no triangle with two right angles in Euclidean or hyperbolic space. We now assume that $K$ is positive.

Without loss of generality we assume

$$
d(q, v) \leq d(p, v)
$$

otherwise we may switch the labels of the vertices $p$ and $q$ (the only feature of the vertex needed is that the angle at that vertex is $\left.\frac{\pi}{2}\right)$. Now, compare the right geodesic triangle $T(p, q, v)$ to the right triangle $T^{\prime}\left(p^{\prime}, q^{\prime}, v^{\prime}\right)$, in the 2-sphere of constant curvature $K$. Since at $p^{\prime}$ the angle between the sides of the triangle $T^{\prime}$ is $\frac{\pi}{2}$ and $d(p, q)=d^{\prime}\left(p^{\prime}, q^{\prime}\right), d(p, v)=d^{\prime}\left(p^{\prime}, v^{\prime}\right)$, the spherical law of cosines radius implies

$$
d^{\prime}\left(p^{\prime}, v^{\prime}\right)<d^{\prime}\left(q^{\prime}, v^{\prime}\right) .
$$

However, this yields a contradiction, as

$$
d(p, v)=d^{\prime}\left(p^{\prime}, v^{\prime}\right)<d^{\prime}\left(q^{\prime}, v^{\prime}\right) \leq d(q, v) \leq d(p, v),
$$

where the second inequality is a consequence of Toponogov's theorem cited above, and the last inequality is (5.1). 
5.2. Background on characteristic foliations. Recall that an oriented singular (this adjective will be implicit in the following) foliation on an oriented surface $S$ is an equivalence class of 1 -forms where $\alpha \sim \beta$ if there is a positive function $f$ such that $\alpha=f \beta$. Let $\alpha$ be a representative for a singular foliation $\mathscr{F}$. A singularity of $\mathscr{F}$ is a point where $\alpha$ vanishes. The singularity $p$ is said to have non-zero divergence if $(d \alpha)_{p}$ is an area form on $T_{p} S$. If $\omega$ is an area form on $S$ (compatible with the chosen orientation), then to each singular point $p$ we attach the sign of the unique real number $\mu$ such that $(d \alpha)_{p}=\mu \omega_{p}$. One can easily check that singular points and their signs do not depend on the choice of $\alpha$ in its equivalence class or of $\omega$ if we keep the same orientation.

Let $S$ be an oriented surface in a contact manifold $(M, \xi)$ with $\xi=\operatorname{ker} \alpha$, cooriented by $\alpha$. The characteristic foliation $\xi S$ of $S$ is the equivalence class of the restriction of $\alpha$ to $S$. The contact condition ensures that all singularities of characteristic foliations have non-zero divergence and hence have non-zero sign. Singularities of $\xi S$ correspond to points where $S$ is tangent to $\xi$ and they are positive or negative according as the orientation of $\xi$ and $S$ match or do not match. We also notice that $\alpha$ provides a co-orientation, and hence if $S$ is oriented by an area form $\omega$ the orientation of the line field $\xi S$ is given by the vector field $X$ which satisfies $\iota_{X} \omega=\left.\alpha\right|_{S}$. One may dually think of the characteristic foliation on $S$ as coming from the singular line field on $S$ given by $T_{p} S \cap \xi_{p}$ for each $p \in S$.

5.3. Characteristic foliations on tori and contact embeddings. We will need to show, informally speaking, how a contact structure which is transverse to the core of the solid torus and "does not rotate more than half a turn between the core and the boundary" embeds inside the standard contact structure on $\mathbb{R}^{3}$. In the following we make this statement precise and provide a proof of it. We will denote by $T$ a torus and by $T_{t}$ the torus $T \times\{t\}$ in the thickened torus $T \times[0,1]$.

We first recall some notions about suspensions on tori. A non-singular foliation $\mathcal{F}$ on $T$ is called a suspension if there is a simple closed curve intersecting all leaves transversely. The name comes from the fact that $\mathcal{F}$ can be reconstructed by suspending the Poincaré first return map on the transversal curve. To such a foliation one can associate a line in $H_{1}(T ; \mathbb{R})$. This line $d(\mathcal{F})$ is called the asymptotic direction of $\mathcal{F}$. We briefly sketch the construction. Pick any point $x$ in $T$, follow the leaf of $\mathcal{F}$ through $x$ for a length $T$, and create a closed curve $O(x, T)$ using a geodesic (for some auxiliary metric). Then the limit homology class $\lim _{T \rightarrow \infty} \frac{1}{T}[O(x, T)]$ exists for every $x$ and it defines a line in $H_{1}(T ; \mathbb{R})$ that does not depend on $x$ or $T$. The limit is called the asymptotic direction. Two easy examples are when $\mathcal{F}$ is linear (we recover the intuitive notion of direction) and when there is a closed leaf (its asymptotic direction is spanned by the homology class of this leaf).

Let $T \times[0,1]$ be a thickened torus. If the characteristic foliations on all the tori $T_{t}$ induced by some contact structure $\xi$ are suspensions, then the contact condition forces the asymptotic directions $d\left(\xi T_{t}\right)$ to always rotate continuously in the same direction (which is determined by the orientations of the manifold, the contact structure and the tori). This direction can be constant along some sub-intervals 1 but it cannot be constant in a neighborhood of $t$ if $\xi T_{t}$ is linear.

If a contact structure $\xi$ on a solid torus $W$ is transverse to a core curve $K$ of $W$, then it lifts to a contact structure on the toric annulus $T \times[0,1]$ obtained

\footnotetext{
${ }^{1}$ This obviously happens around each $t$ such that $\xi T_{t}$ is structurally stable.
} 
by blowing 2 up $K$. The lifted contact structure induces a linear foliation on the boundary component which projects to $K$, say $T_{0}$. The direction of this foliation is spanned by the meridian class, i.e. the class in $H_{1}(T)$ which spans the kernel of the map from $H_{1}(T \times[0,1])$ to $H_{1}(W)$ induced by the projection.

The following lemma gives a precise formulation of the idea described informally at the beginning of this subsection.

Lemma 5.4. Let $\xi^{\prime}$ be a contact structure on a solid torus $W$ transverse to a core curve $K$ of $W$. Let $\xi$ be the contact structure on $T \times[0,1]$ lifted from $W$ as described above. If all characteristic foliations $\xi T_{t}$ are suspensions whose asymptotic directions never contain the meridian homology class for $t>0$, then $\left(W, \xi^{\prime}\right)$ is contactomorphic to a domain in $\left(S^{1} \times \mathbb{R}^{2}, \xi_{\text {rot }}=\operatorname{ker}\left(d \phi+r^{2} d \theta\right)\right)$.

This lemma is an easy consequence of the following result of Giroux.

Theorem 5.5 (Giroux [9, Theorem 3.3]). Suppose two contact structures $\xi_{0}$ and $\xi_{1}$ on $T \times[0,1]$ induce suspensions on each torus $T_{t}$ which agree on $T_{0}$ and $T_{1}$. If the two paths of asymptotic directions $t \mapsto d\left(\xi_{i} T_{t}\right)$ are non-constant and homotopic relative to their common end-points, then $\xi_{0}$ and $\xi_{1}$ are isotopic relative to the boundary $T_{0} \cup T_{1}$.

Proof of Lemma 5.4. Because of the asymptotic direction assumption, we can choose a longitudinal curve $L$ for $W$ whose homology class does not belong to the asymptotic direction of any of the foliations $\xi T_{t}$ (pick any longitude and add a sufficiently large multiple of the meridian). In particular we can choose $L$ to intersect transversely all leaves of $\xi \partial W$ and such that the corresponding Poincaré map rotates all points clockwise. So there is an identification of $(W, \partial W)$ with $\left(D^{2} \times S^{1}, S^{1} \times S^{1}\right)$ sending $L$ to some $\{*\} \times S^{1}$ and $\xi \partial W$ to a foliation directed by $v=\frac{\partial}{\partial \theta}+F(\theta, \phi) \frac{\partial}{\partial \phi}$ where we use coordinates $(\theta, \phi)$ on $S^{1} \times S^{1}$ and $F(\theta, \phi) \leq 0$.

We now consider an embedding $\varphi$ of $D^{2} \times S^{1}$ into $\mathbb{R}^{2} \times S^{1}$ which is the identity along $\{0\} \times S^{1}$ and agrees with $(1, \theta, \phi) \mapsto\left((-F(\theta, \phi))^{\frac{1}{2}}, \theta, \phi\right)$ along $S^{1} \times S^{1}$. An immediate computation reveals that $\varphi$ sends $\xi \partial W$ to $\xi_{\text {rot }} \varphi(\partial W)$. Using the standard neighborhood theorem for curves transverse to a contact structure, it can also be easily arranged that $\varphi$ sends $\xi$ to $\xi_{\text {rot }}$ along $K$. One may now blow up $K$ and apply Giroux's theorem to further isotope $\varphi$ to a contact embedding. The homotopy hypothesis is guaranteed because the homology class of $[L]$ belongs to no asymptotic direction of a $\xi T_{t}$.

Proof of Proposition 5.2. The vector field $n_{\mathrm{D}}$ is tangent to each torus $T_{r}=\partial \mathrm{T}(r)$, $0<r \leq r_{0}$, and $\xi$ is transverse to $n_{\mathrm{D}}$ so each characteristic foliation $\xi T_{r}$ is nonsingular and transverse to $n_{\mathrm{D}}$. In addition, $n_{\mathrm{D}}$ is transverse to the foliation by meridian circles of $T_{r}$ coming from the disks $D_{z}$, so it is a suspension. So there is a simple closed curve $\mathcal{C}$ in $T_{r}$ which is not a meridian and transversely intersects all leaves of $\xi T_{r}$ (the homology class of $\mathcal{C}$ belongs to a rational approximation of the asymptotic cycle of $\left.n_{\mathrm{D}}\right)$. For brevity, we denote the asymptotic directions $d\left(\xi T_{r}\right)$ and $d\left(n_{\mathrm{D}}\right)$ by $X$ and $N$, respectively, and by $M$ the line spanned by the meridian homology class. One can see $X$ and $N$ as points on the circle $P\left(H_{1}\left(T^{2} ; \mathbb{R}\right)\right)$ continuously moving as $r$ increases. Note that $X$ initially equals $M$ and moves

\footnotetext{
${ }^{2}$ The knot $K$ has a tubular neighborhood $D_{\varepsilon}^{2} \times S^{1}$ with coordinates $\left(r e^{i \theta}, \varphi\right)$ such that $\xi=$ ker $d \varphi+r^{2} d \theta$. The blow-up map from $[0,1] \times S^{1} \times S^{1}$ to $D^{2} \times S^{1}$ is $\operatorname{simply}(s, \theta, \varphi) \mapsto\left(\sqrt{s} e^{i \theta}, \varphi\right)$ on $\left[0, \varepsilon^{2}\right] \times S^{1} \times S^{1}$ and interpolated to $(s, \theta, \varphi) \mapsto\left(s e^{i \theta}, \varphi\right)$ on $[\varepsilon, 1] \times S^{1} \times S^{1}$.
} 
clockwise in a monotone way, whereas $N$ moves in some way but never hits $M$ or $X$. This easily implies that $X$ cannot become meridional and one can apply Lemma 5.4 to obtain the desired conclusion.

In order to prove Proposition 5.3 we first need to understand neighborhoods of disks transverse to Reeb vector fields.

Lemma 5.6. Let $M$ be a 3-manifold with a contact form $\alpha$ and $D$ a closed disk embedded in $M$. If the Reeb vector field of $\alpha$ is transverse to $D$, then there is a neighborhood $V$ of $D$ in $M$ and an embedding $\varphi$ of $V$ in $\mathbb{R}^{3}$ such that $\varphi(D)$ is transverse to vertical lines in $\mathbb{R}^{3}$ and $\varphi_{*} \alpha=d z+r^{2} d \theta$.

Proof. Let $D^{\prime}$ be a disk containing $D$ and still transverse to the Reeb vector field $R$. Let $\varepsilon$ be a positive number such that the flow of $R_{\alpha}$ embeds $D^{\prime} \times[-\varepsilon, \varepsilon]$ into $M$. If $r$ and $\theta$ are polar coordinates on $D^{\prime}$ and $z$ is the coordinate in $[-\varepsilon, \varepsilon]$, the pull-back of $\alpha$ is $d z+f(r, \theta) d r+g(r, \theta) d \theta$ for some functions $f$ and $g$. This contact form extends trivially to $D^{\prime} \times \mathbb{R}$ as the contactization of the exact symplectic manifold $\left(D^{\prime}, \beta\right)$ where $\beta=f d r+g d \theta$. We now think of $D^{\prime}$ as the unit disk in $\mathbb{R}^{2}$. The following claim will be proved below.

Claim. There is an extension of $\beta$ to $\mathbb{R}^{2}$ such that $d \beta$ is symplectic everywhere and $\beta=r^{2} d \theta$ outside some large disk.

Since the symplectic condition is convex in dimension $2, \alpha_{t}=d z+(1-t) \beta+$ $t r^{2} d \theta, t \in[0,1]$, is a family of contact forms on $\mathbb{R}^{2} \times \mathbb{R}$. We now use a general fact: if $\beta_{t}$ is a family of 1 -forms on a surface $S$ such that each $d \beta_{t}$ is symplectic and all $\beta_{t}$ agree outside some compact set, then there is an isotopy $\varphi_{t}$ of the contactization $S \times \mathbb{R}$ sending surfaces $S \times\{z\}$ to surfaces transverse to lines $\{s\} \times \mathbb{R}$ and such that $\varphi_{t}^{*} \alpha_{t}=\alpha_{0}$. The isotopy is constructed using Moser's technique as the flow of a vector field $X_{t}=Y_{t}+\lambda_{t} \partial_{z}$ with $Y_{t}$ the vector field on the surface defined by $d \beta_{t}\left(Y_{t}, \cdot\right)=-\dot{\beta}_{t}$ and $\lambda_{t}=-\beta_{t}\left(Y_{t}\right)$. The transversality condition comes from the commutation of $X_{t}$ and $\partial_{z}$.

We now prove the claim. First we fix the symplectic form $\omega=r d r \wedge d \theta$ on $\mathbb{R}^{2}$ so that the problem is reformulated in terms of vector fields $\omega$-dual to the forms we consider. We have a vector field $Y$ with positive divergence on $D^{\prime}$ and we want to extend it to $\mathbb{R}^{2}$ such that it coincides with $Y_{0}=r \partial_{r}$ outside some large disk. We denote by $D_{r}$ the disk of radius $r$ around the origin so $D^{\prime}=D_{1}$. We first extend $Y$ arbitrarily to a neighborhood of $D_{1}$ so its divergence stays positive in some $D^{\prime \prime}=D_{1+\delta}$. Let $h$ be a smooth function from $[0, \infty)$ to $\mathbb{R}$ with $h$ and $h^{\prime}$ vanishing on $[0,1]$ and everywhere non-negative. If $h$ grows sufficiently fast between 1 and $1+\delta$, the vector field $Y^{\prime}=Y+h \partial_{r}$ has positive divergence and is transverse to the boundary of $D^{\prime \prime}$. It is then easy to extend $\left.Y^{\prime}\right|_{D^{\prime \prime}}$ to a vector field which is transverse to all circles $\partial D_{r}$ for $r \geq 1+\delta$ and has the same orbits as $Y_{0}$ outside the disk $D_{1+2 \delta}$. We can then rescale it in the region between $D^{\prime \prime}$ and some large disk $D_{r}$ (here we do not control $r$ ) so that it still has positive divergence and coincides with $Y_{0}$ outside $D_{r}$.

Proof of Proposition 5.3 . Since any ball inside $\left(S^{1} \times \mathbb{R}^{2}, \operatorname{ker}\left(d \phi+\rho^{2} d \theta\right)\right)$ easily embeds inside $\left(\mathbb{R}^{3}, \xi_{\text {std }}\right)$ we only need to construct an embedding into the former model. The proposition will follow from the construction of an embedding of $\mathrm{T}(r)$ into a solid torus satisfying the hypothesis of Lemma 5.4. We construct this embedding in several steps. 
We first introduce a technical definition. Let $Y$ be either a closed interval or $S^{1}$. Let $D^{2}$ be the unit disk in $\mathbb{R}^{2}$ and let $D_{r}$ denote the disk of radius $r$. A contact structure on a $D^{2} \times Y$ is under control if there is a vector field tangent to $\{0\} \times Y$ and to $\partial D_{r} \times Y$ for all $r$ which is transverse to both $\xi$ and the obvious foliation by disks. A contact structure on a domain $C$ is under control if there is a diffeomorphism from $C$ to $D^{2} \times Y$ sending $\xi$ to a contact structure under control. The inverse images of the objects involved in the above discussion are then said to control $\xi$. In particular, in our geometric setup, $\xi$ is under control on $\mathrm{T}(r)$ and Lemma 5.4 applies to any contact structure which is under control on a solid torus.

We want to construct thickened disks $T_{t}$ and $T_{b}$ that can be glued to the top and bottom, respectively, of $\mathrm{T}(r)$ so that the characteristic foliation on the top and bottom of $U=\mathrm{T}_{t} \cup \mathrm{T}(r) \cup \mathrm{T}_{b}$ is "standard" and $\xi$ stays under control on this larger thickened disk. (Here top and bottom refer to $\mathrm{T}(r)$ seen with $\zeta$ vertical and oriented from bottom to top. Also "standard" means that the singular foliation has a single elliptic singularity and the rest of the leaves are radial.) Thus we will be able to glue the top and bottom of $U$ together to obtain a solid torus $S$ with a contact structure under control into which T $(r)$ embeds, and Lemma 5.4 will finish the proof.

We discuss the construction of $\mathrm{T}_{t}$, the construction of $\mathrm{T}_{b}$ being analogous. Let $z_{t}$ be the top extremity of $\zeta$ and $\mathrm{D}:=\mathrm{D}_{z_{t}}$. The previous lemma gives a contact embedding $\varphi$ of a neighborhood of $D$ in $\mathbb{R}^{2} \times \mathbb{R}$ such that the image of $D$ is the graph of some function $f$ over some (deformed) disk $\Omega$ in $\mathbb{R}^{2}$.

Let $K$ be a constant such that $f(\rho, \theta)<K$ for all $(\rho, \theta) \in \Omega$. Set $C=$ $\{(\rho, \theta, z) \mid(\rho, \theta) \in \Omega, f(\rho, \theta) \leq z \leq K\}$; this will (almost) be the bottom part of $\mathrm{T}_{t}$. It is foliated by the graphs $G_{s}$ of functions $(1-s) f+s K, s \in[0,1]$, and can also be seen as the union of the Reeb orbit $\varphi\left(z_{t}\right) \times\left[f\left(z_{t}\right), K\right]$ and vertical annuli over the images $c_{r}$ of the $\partial \mathrm{T}(r) \cap \mathrm{D}$. Together with the vector field $\partial_{z}$, these objects show that $\xi_{\text {std }}$ is under control on $C$. We now smooth $\varphi(\mathrm{T}(r) \cap \operatorname{Dom}(\varphi)) \cup C$ to get a thickened disk $C^{\prime}$ extending $\varphi(\mathrm{T}(r) \cap \operatorname{Dom}(\varphi))$ above $\varphi(D)$ which coincides with $C$ when $z$ is close to $K$ and such that $\xi_{\text {std }}$ is under control on $C^{\prime}$.

Then we notice that there is a large number $R$ such that $\Omega$ is contained in the disk of radius $R$ about the origin and there is an isotopy $\Psi_{t}: \Omega \rightarrow \mathbb{R}^{2}, t \in[0,1]$, such that $\Psi_{0}=i d_{\Omega}$ and $\Psi_{1}(\Omega)$ is a disk of radius $R$ centered about the origin. Consider the embedding $\Psi: \Omega \times[0,1] \rightarrow \mathbb{R}^{3}$ defined by $\Psi(p, t)=\left(\Psi_{t}(p), K+K^{\prime} t\right)$, where $K^{\prime}$ is a large positive constant to be determined soon. For each radius $r$, the characteristic foliation on $\Psi\left(c_{r} \times[0,1]\right)$ is given as the kernel of $\left.\Psi\right|_{c_{r} \times[0,1]} ^{*}\left(d z+\rho^{2} d \theta\right)=K^{\prime} d t+\beta$, where $\beta$ is independent of $K^{\prime}$. Thus for $K^{\prime}$ large enough $\Psi_{*} \frac{\partial}{\partial t}$ is never tangent to the characteristic foliation of $\Psi\left(c_{r} \times[0,1]\right)$ and $\xi_{\text {std }}$ is under control on the image of $\Psi$. We then choose $\mathrm{T}_{t}$ to be a smoothed version of $C^{\prime} \cup \Psi(\Omega \times[0,1])$. It can be glued to $\mathrm{T}(r)$ using $\varphi$. After doing the same thing for the bottom of $\mathrm{T}(r)$ we get $\mathrm{T}_{b}$ and we can do the construction of $\mathrm{T}_{b}$ and $\mathrm{T}_{t}$ with the same large radius $R$ so that the top and bottom of $\mathrm{T}_{b} \cup \mathrm{T}(r) \cup \mathrm{T}_{t}$ can be glued to get a solid torus with a contact structure under control.

\section{ACKNOWLEDGMENTS}

The authors wish to thank Vladimir Krouglov for useful correspondence and Yasha Eliashberg for pointing out the fillability of integrable CR manifolds. They also thank the referee for pointing out several potential pitfalls and providing highly 
valuable input that improved the paper. The second author is grateful to Chris Croke for enjoyable lunch meetings and many helpful suggestions to the project. The first author was partially supported by the NSF Grant DMS-0804820 and DMS1309073. The second author was partially supported by DARPA YFA-N66001-111-4132. The third author was partially supported by the ANR grant ANR-10-JCJC 0102 .

\section{REFERENCES}

[1] Peter Albers and Helmut Hofer, On the Weinstein conjecture in higher dimensions, Comment. Math. Helv. 84 (2009), no. 2, 429-436, DOI 10.4171/CMH/167. MR2495800 (2010b:53152)

[2] Daniel Bennequin, Entrelacements et équations de Pfaff (French), Third Schnepfenried geometry conference, Vol. 1 (Schnepfenried, 1982), Astérisque, vol. 107, Soc. Math. France, Paris, 1983, pp. 87-161. MR753131 (86e:58070)

[3] David E. Blair, Riemannian geometry of contact and symplectic manifolds, Progress in Mathematics, vol. 203, Birkhäuser Boston, Inc., Boston, MA, 2002. MR.1874240 (2002m:53120)

[4] Matthew Strom Borman, Yakov Eliashberg and Emmy Murphy, Existence and classification of overtwisted contact structures in all dimensions. Preprint 2014.

[5] Isaac Chavel, Riemannian geometry: A modern introduction, 2nd ed., Cambridge Studies in Advanced Mathematics, vol. 98, Cambridge University Press, Cambridge, 2006. MR2229062 (2006m:53002)

[6] Kai Cieliebak and Yakov Eliashberg, From Stein to Weinstein and back: Symplectic geometry of affine complex manifolds, American Mathematical Society Colloquium Publications, vol. 59, American Mathematical Society, Providence, RI, 2012. MR3012475

[7] Yakov Eliashberg, Contact 3-manifolds twenty years since J. Martinet's work (English, with French summary), Ann. Inst. Fourier (Grenoble) 42 (1992), no. 1-2, 165-192. MR.1162559 (93k:57029)

[8] John B. Etnyre, Rafal Komendarczyk, and Patrick Massot, Tightness in contact metric 3-manifolds, Invent. Math. 188 (2012), no. 3, 621-657, DOI 10.1007/s00222-011-0355-2. MR2917179

[9] Emmanuel Giroux, Structures de contact en dimension trois et bifurcations des feuilletages de surfaces (French), Invent. Math. 141 (2000), no. 3, 615-689, DOI 10.1007/s002220000082. MR 1779622 (2001i:53147)

[10] Alfred Gray, Tubes, 2nd ed., Progress in Mathematics, vol. 221, Birkhäuser Verlag, Basel, 2004. With a preface by Vicente Miquel. MR2024928 (2004j:53001)

[11] R. E. Greene and H. Wu, On the subharmonicity and plurisubharmonicity of geodesically convex functions, Indiana Univ. Math. J. 22 (1972/73), 641-653. MR0422686 (54 \#10672)

[12] H. Hofer, Pseudoholomorphic curves in symplectizations with applications to the Weinstein conjecture in dimension three, Invent. Math. 114 (1993), no. 3, 515-563, DOI 10.1007/BF01232679. MR 1244912 (94j:58064)

[13] Hermann Karcher, Riemannian comparison constructions, Global differential geometry, MAA Stud. Math., vol. 27, Math. Assoc. America, Washington, DC, 1989, pp. 170-222. MR1013810 (91b:53046)

[14] Patrick Massot, Klaus Niederkrüger, and Chris Wendl, Weak and strong fillability of higher dimensional contact manifolds, Invent. Math. 192 (2013), no. 2, 287-373, DOI 10.1007/s00222012-0412-5. MR 3044125

[15] Klaus Niederkrüger, The plastikstufe - a generalization of the overtwisted disk to higher dimensions, Algebr. Geom. Topol. 6 (2006), 2473-2508, DOI 10.2140/agt.2006.6.2473. MR.2286033(2007k:57053)

[16] Klaus Niederkrüger and Federica Pasquotto, Resolution of symplectic cyclic orbifold singularities, J. Symplectic Geom. 7 (2009), no. 3, 337-355. MR2534189 (2010j:53176)

[17] Peter Petersen, Riemannian geometry, 2nd ed., Graduate Texts in Mathematics, vol. 171, Springer, New York, 2006. MR2243772(2007a:53001)

[18] Bruce L. Reinhart, The second fundamental form of a plane field, J. Differential Geom. 12 (1977), no. 4, 619-627 (1978). MR512930(80a:57013) 
[19] Philippe Rukimbira, Chern-Hamilton's conjecture and K-contactness, Houston J. Math. 21 (1995), no. 4, 709-718. MR.1368340 (96m:53032)

[20] Takashi Sakai, Riemannian geometry, Translations of Mathematical Monographs, vol. 149, American Mathematical Society, Providence, RI, 1996. Translated from the 1992 Japanese original by the author. MR.1390760 (97f:53001)

School of Mathematics, Georgia Institute of Technology, Atlanta, Georgia 30332 E-mail address: etnyre@math.gatech.edu

$U R L:$ http://www.math.gatech.edu/ etnyre/

Department of Mathematics, Tulane University, New Orleans, Louisiana 70118

E-mail address: rako@tulane.edu

URL: http://www.math.tulane.edu/ rako/

Département de Mathématiques, Université Paris Sud, 91405 Orsay Cedex, France Current address: Centre de Mathématiques Laurent Schwartz, École Polytechnique, 91128 Palaiseau Cedex, France

E-mail address: patrick.massot@polytechnique.edu 